\title{
Erratum to: Neogene eastern Amazon carbonate platform and the paleoenvironmental interpretation
}

\author{
Orangel Aguilera · José Tasso Felix Guimarães • \\ Heloisa Moraes-Santos
}

Received: 15 October 2012/ Accepted: 8 February 2013

(C) Akademie der Naturwissenschaften Schweiz (SCNAT) 2014

\begin{abstract}
The Early Miocene Pirabas Formation represents extensive carbonate and siliciclastic belts deposited in marine coastal environments along the eastern Amazon coast. This formation was studied in its palynological, crustacean decapods and sedimentary facies, to develop a model of its depositional processes, faunal and floral assemblages and the sedimentary environments, as observed in the B17 mine (Capanema, Pará). The sedimentary records consist mostly of light gray to greenish gray mudstones with tidal bedding, calciferous sandstones, stratified biocalcirudites and levels with conglomerates deposited in a carbonate/siliciclastic transitional system with lagoon, tidal flat, flood-tide delta and foreshore environments. 18 palynomorph species were identified in the tidal flat deposits including one alga, five pteridophytes and nine angiosperms. Among the latter, mangrove pollen and spores of Zonocostites ramonae, Zonocostites minor
\end{abstract}

This article has been published before under doi:10.1007/s13358-0130051-5. Unfortunately, it has been an uncorrected version which should not be cited. For future citations, please refer to this corrected version under doi:10.1007/s13358-013-0066-6.

The online version of the original article can be found under doi:10.1007/s13358-013-0051-5.

O. Aguilera $\cdot$ H. Moraes-Santos

Museu Paraense Emilio Goeldi, Coordenação de Ciências da

Terra e Ecologia, CCTE, Av. Perimetral, 1901, Terra Firme,

Belém, PA 66077-830, Brazil

e-mail: orangel.aguilera@gmail.com

H. Moraes-Santos

e-mail: hmoraes@museu-goeldi.br

J. T. F. Guimarães ( $₫)$

Instituto Tecnológico Vale-ITV, Rua Boa Ventura da Silva, $\mathrm{n}^{\circ}$ 955, $3^{\circ}$ andar, Umarizal, Belém, PA 66055-090, Brazil

e-mail: tasso.guimaraes@itv.org and Deltoidospora adriennis are reported. Nine species of crustacean decapods were recognized in the foreshore deposits, comprising one Callianassoidae, two Calappidae and six Portunidae, represented by Euphylax, $\uparrow$ Necronectes, Portunus and Scylla, the ecology of which is associated with beaches of marginal lagoons. The main factor in the formation of these carbonate environments is assumed to have been related to the lack of a large-scale drainage system such as the Amazon River basin in the transition Oligocene-Miocene, which may also have influenced carbonate production and resulted in a strong decrease of paleodischarges from incipient river systems along the Amazon coast.

Keywords Amazon - Carbonate - Pirabas · Paleoenvironment $\cdot$ Palynology $\cdot$ Crustacea

\section{Introduction}

The tidal shelf of the eastern Amazon was characterized by an extensive and thick accumulation of early Miocene carbonate deposits related to the Pirabas Formation (Maury 1925), the latest carbonate deposit in northern Brazil. The Amazon fan was only incipient during late Oligoceneearly Miocene and the influence of siliciclastic sediments supplied by the river flow to the Atlantic coast was low (Figueiredo et al. 2009), which has been attributed to the initial eastern Andean cordillera uplift (Shephard et al. 2010) during the Oligocene (Horton et al. 2010). Therefore, the substantial Andean uplift in the late Miocene resulted in the establishment of the Amazon drainage to the Atlantic coast (Hoorn et al. 1995). The low sediment input into the ocean during early Miocene favored carbonate formation and expansion of the photic zone, and the development of a 
high-diversity shallow marine flora and fauna along the eastern Amazon coast.

The sedimentary deposition of the Pirabas Formation was influenced by water masses with high-energy flow of the Atlantic Ocean (Johns et al. 1998, 2002), with longshore currents driven by oceanic currents, a macrotidal regime (Beardsley et al. 1995) and wave processes. Additionally, changes in the sources of siliciclastic sediments, the water temperature (Zachos et al. 2001), and the eustatic and relative sea-level oscillations (Haq et al. 1988; Carter 1998; Kominz et al. 2008) controlled the distribution of carbonate and siliciclastic belts deposited in the marine coastal environments (Rossetti 2006; Rossetti and Santos 2004).

The heterotrophic carbonate producers, such as mollusks, echinoids and bryozoans, were abundant and diverse in the Pirabas Formation (Rossetti and Góes 2004; Távora et al. 2010; Aguilera and Páes 2012). Oligotrophic corals are absent, except for isolated records of Agariciidae (Lalor and Távora 2006). The early Miocene Pirabas Formation is time equivalent to the period when the Caribbean reefbuilding corals had declined and the diversity became reduced by $50 \%$ (Johnson et al. 2009). Similarly, Bourrouilh-Le Jan and Hottinger (1988) described a drastic early Miocene decline in coral reefs on a number of Pacific atolls. This absence of corals in the tropics with respect to carbonate producers may correspond to the worldwide bloom of coralline red-algal (rhodalgal) facies (Halfar and Mutti 2005).

Many plant remains were also identified along the coastal deposits of the Pirabas Formation (Toledo 1989; Arai 1997; Leite et al. 1997a, b; Fernandes 1984; Távora et al. 2010), suggesting deposition in a tidal flat and lagoon environments (Rossetti 2006; Rossetti and Santos 2004). Leite et al. (1997a, b) proposed that the paleovegetation of the Pirabas Formation is marked by the predominance of Zonocostites ramonae Gemeraad et al. 1968, a Miocene species that inhabited mangrove-type environments.

There are at least 27 outcrop localities recorded from the Pirabas Formation (Távora et al. 2010). However, the exposures are restricted in thickness to a few meters along the tidal zone. The only exception is the Capanema Mine B17, with around $40 \mathrm{~m}$ thick of exposed section. Here, we recorded an exceptional horizon with hundreds of crab carapaces, chelae and fragments of portunids (blue crabs), calappids (box crabs) and suitable palynological samples for a paleoenvironmental study.

Early contributions of White (1887) and Maury (1925), particularly on mollusk, bryozoan and coral research, and Petri (1954, 1957) on foraminifera, Beurlen (1958), Brito (1971, 1972, 1977) and Martins-Neto (2001) on crustaceans, Santos $(1958,1967)$ on echinoids, Santos and Travassos (1960) and Santos and Salgado (1971) on fishes,
Paula-Couto (1967), Toledo (1989), and Toledo and Domning (1989) about sirenians, and Lalor and Távora (2006) on corals, as well as many further references summarized in Rossetti and Góes (2004) and in Távora et al. (2010), provide additional descriptions, records and/or reviews of different fossils groups from the Pirabas Formation.

Considering the regional relevance of this fossiliferous carbonate deposit in relation to the Atlantic Ocean, the objective of this paper is the integrated study of palynology, crustacean decapods and facies research for the interpretation of the paleoenvironment.

\section{Geological setting}

The Bragantina platform (North Brazil, Pará) corresponds to emerged and submerged areas, which were stable during episodes of distention tectonics following the breakup of Gondwana and the opening of the Equatorial Atlantic during Jurassic and Lower Cretaceous (Szatmari et al. 1987). Despite this tectonic stability, thick sedimentary units of Miocene age were deposited in this region. Therefore, Rossetti (2001) proposed that the reactivation of normal and strike-slip faults from Precambrian basement, combined with sea-level changes, was responsible for the genesis of these units in the deep tectonic troughs (e.g., Vigia-Castanhal and Marajó basins). The Neotectonic activity of the northeastern Pará is controlled by E-W transcurrent faults connected with NW-SE normal faults, while the structural and sedimentary evolution is related to the reactivation of the basement architecture (Costa et al. 1996). Structural entities such as the Pará platform and Bragança-Viseu basin are shown in Fig. 1a.

The Bragantina platform comprises around 2,000 $\mathrm{m}$ of Jurassic/Cretaceous deposits. The sedimentary cover of the Paleogene and Neogene ( $\sim 1,500 \mathrm{~m}$ thick) is not tectonically influenced by the ancient depositional centers, which indicates higher stability in the adjacent coastal basins (Gorini and Bryan 1976). The Bragança-Viseu basin represents a Graben bordered by normal faults along a NWSE structural profile. The sediments are composed of litostratigraphic units of Cretaceous age, including the Bragança, Grajaú, Codó and Itapecuru formations, which are overlain by Neogene carbonate rocks of the early Miocene Pirabas Formation, which in turn is overlain by the siliciclastic Miocene Barreiras Group and the Pleistocene Pós-Barreiras Formation (Figs. 1b, 2).

The early Miocene Pirabas Formation (Maury 1925) consists of carbonate rocks of an offshore shelf environment (grainstone and consolidated packstone, stratified wackestone packstone and laminated mudstone). However, coastal facies (shoreface/foreshore), marginal lagoons, 

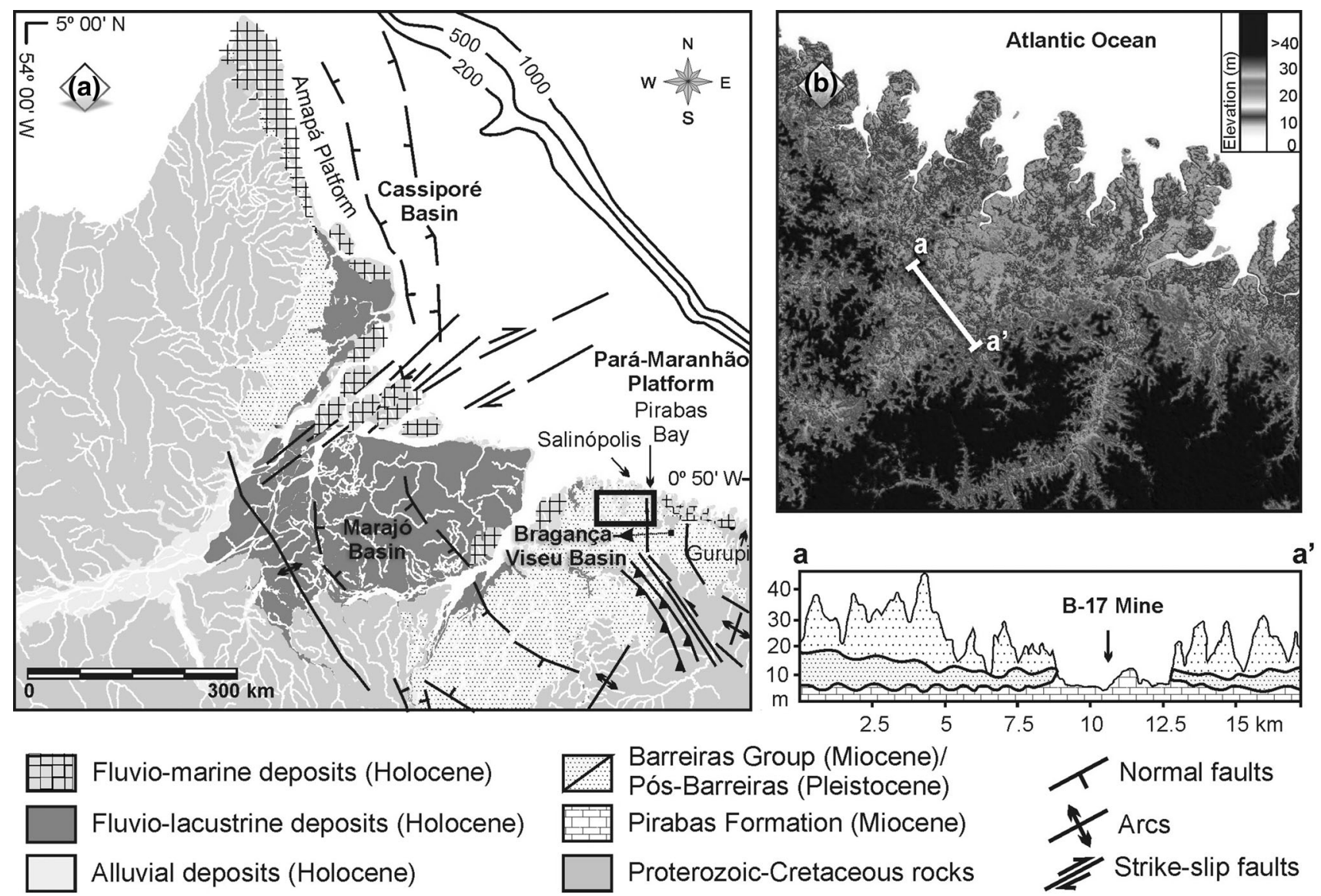

Fluvio-marine deposits (Holocene)

Fluvio-lacustrine deposits (Holocene) Alluvial deposits (Holocene)

Barreiras Group (Miocene)/ Pós-Barreiras (Pleistocene) Pirabas Formation (Miocene)
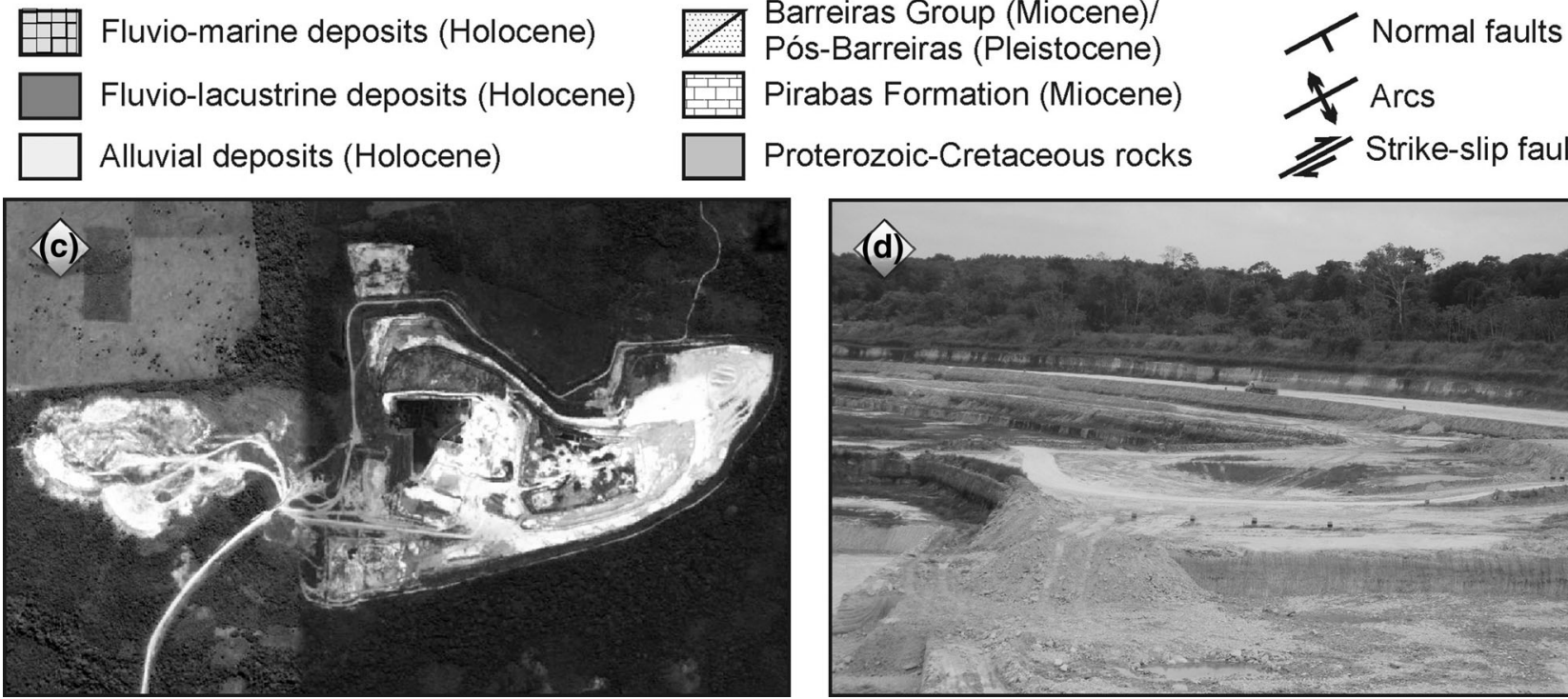

Proterozoic-Cretaceous rocks
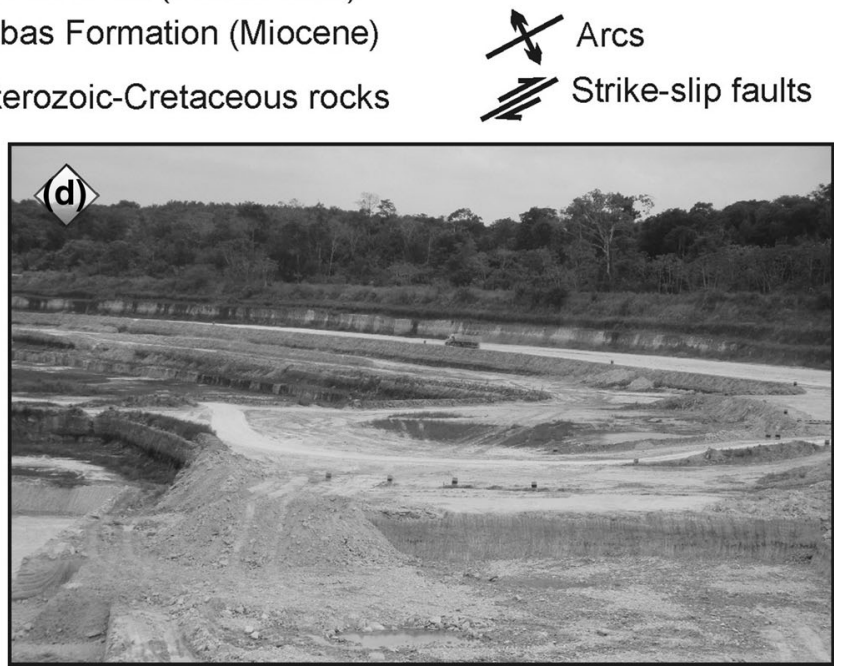

Fig. 1 Location map. a Regional geology. b Digital elevation model of the northern coast of the Pará state. c Aerial view (modified from Google earth) and $\mathbf{d}$ panoramic view of the B17 mine

(gray to olive mudstone and conglomeratic sandstone) and tidal flats (massive dark mudstone) have also been recorded (Góes et al. 1990; Rossetti 2001; Rossetti and Góes 2004). More details of these heterogeneous lithostratigraphic sequences and the associated fossil faunas were presented and discussed by Aguilera and Páes (2012).

The assignment of early Miocene for the Pirabas Formation follows Petri (1957), Fernandes (1984), Ferreira et al. (1978), Fernandes and Távora (1990) and Távora and Fernandes (1999). The heterogeneous variation of the faunal composition recorded in the different outcrops (Aguilera and Páes 2012) suggests that different members should be recognized, and accurate geochronological research may reveal different ages within the Pirabas sequence.

\section{Materials and methods}

Several field trips (August and December 2011 and April 2012) to the B17 mine of the CIBRASA S/A near 
Fig. 2 Stratigraphic chart for the Bragantina Platform, Brazil (modified from Rossetti 2001)

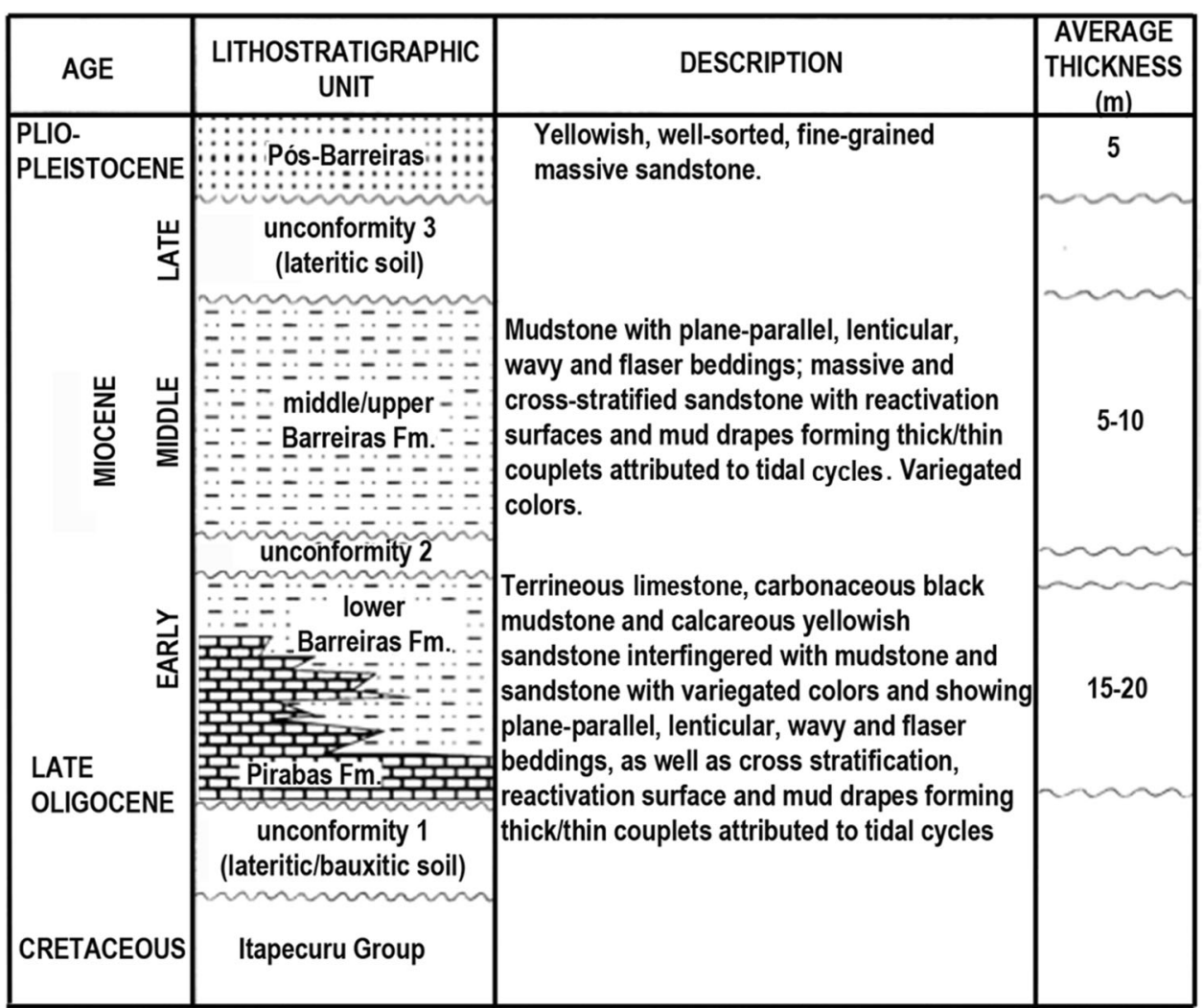

Capanema, Pará (Fig. 1c, d; $01^{\circ} 10^{\prime} \mathrm{S}, 47^{\circ} 13^{\prime} \mathrm{W}$ ) permitted the construction of a biostratigraphic section and facies interpretation, based on direct field measurements and analysis. Facies analysis included descriptions of color, lithology, texture and structures based on methods presented by Walker (1992), and were codified according to Miall (1978).

Four bulk samples were collected from dark gray mudstones rich in organic matter of the section intervals between 9-8 and 5-4 $\mathrm{m}$ for palynological analysis (Fig. 3). Around $30 \mathrm{~g}$ per sample was dried, weighed, triturated, submitted to hydrochloric and hydrofluoric acid for carbonate and silicate removal, sieved with a $10 \mu \mathrm{m}$ mesh and mounted on glass slides with Entelan medium following Uesugui (1979). Pollen and spore morphologies are based on Traverse (2008) and Hesse et al. (2008). The South American palynomorphs references are based on a rich existing literature (e.g., Van der Hammen 1956a, b; Van der Hammen and Wymstra 1964; Germeraad et al. 1968; Dueñas 1980; Lorente 1986; Muller et al. 1987; Hoorn 1993, 1994a, b; Jaramillo and Dilcher 2001; SilvaCaminha et al. 2010). Pollen and spores were described using the terminology of Punt et al. (2007) and the classification system of Iversen and Troels-Smith (1950). The pollen and spore taxonomic affinities and ecological interpretation follow Roubik and Moreno (1991),
Colinvaux et al. (1999) and Bush and Weng (2006). Samples were counted to a minimum of about 300 pollen grains. Pollen and spore data are presented in pollen diagrams as percentages of the total pollen amount. Taxa were grouped into mangrove, lowland freshwater forests, lowland pteridophytes, fungi, algae and foraminifera. The softwares Tilia and Tilia Graph were used for the calculation and plotting of palynological diagrams (Grimm 2004).

Fossil macro invertebrates, particularly crustacean decapods, were collected directly from the surface of the calciferous sandstone interval of 10-9 m during ongoing mining activities. The study of the fossil crustacean decapods was based on comparative analysis, with the collections from the Museu Paraense Emilio Goeldi, the Universidad Experimental Francisco de Miranda (see Aguilera et al. 2011) and those deposited at the Smithsonian Tropical Research Institute. Collections from the Pirabas Formation at the Museu de Geologia in the Universidade Federal do Pará, under the care of V. Távora, could not be accessed. Complementary data were retrieved from published literature (e.g., Beurlen 1958; Brito 1971, 1972, 1993; Martins-Neto 2001, 2005; Martins-Neto and Dias-Júnior 2007; Vega et al. 2009; Schweitzer et al. 2006). The classification follows De Grave et al. (2009) and Schweitzer et al. (2009). 
Fig. 3 Stratigraphic section of the B17 mine. 1 Facies, $M m$ massive mudstone, $\mathrm{Hl}$ lenticular heterolithic, $B c$ biocalcirudite with cross-stratification, $\mathrm{Bm}$ massive biocalcirudite, $S p$ sandstone with planar and/or low-angle cross-stratification, $\mathrm{Hi}$ inclined heterolithic, Sca calciferous sandstone. 2 Facies association, $L G$ lagoon, $T F$ tidal flat, $F D$ flood-tide delta, $F S$ foreshore

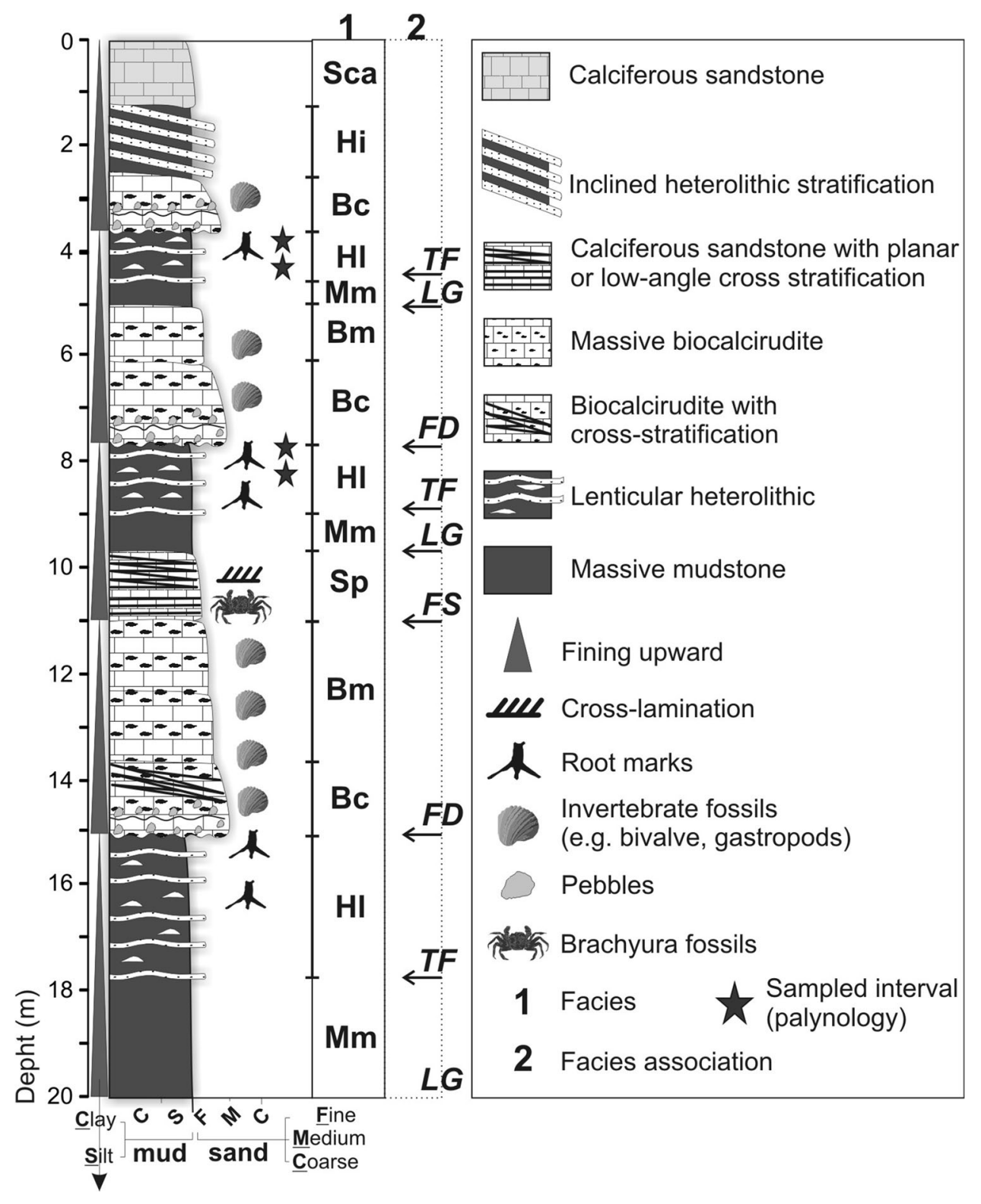

\section{Results}

Facies description from the Capanema Mine B17 section

The sedimentary record consists mostly of light gray to greenish gray mudstones with tidal bedding, calciferous sandstones, stratified biocalcirudites and levels with conglomerates. Invertebrates, pollen and spore records were added to facies' characteristics to define four facies associations grouped into fining and thinning upward cycles that represent a carbonate/siliciclastic transitional system, with lagoon (LG), tidal flat (TF), flood-tide delta (FD) and foreshore (FS) environments (Fig. 3; Table 1).
Lagoon $(L G)$. This association is represented by massive mudstones without apparent structures. It is laterally continuous (facies $\mathrm{Mm}$ ), about $2 \mathrm{~m}$ thick in the basal strata and decreases to $0.5 \mathrm{~m}$ along the sedimentation cycles (Fig. 3; Table 1). The LG association corresponds to a low-energy environment associated with the basin center and a deposition of mud from the suspension.

Tidal flat (TF). The TF association occurs at the top of each sedimentary cycle and represents mud with connected and discontinuous lenses of rippled sand (facies $\mathrm{Hl}$ ). Locally, root fragments and root marks can be observed. In the upper strata, the base of this facies is erosive, containing biocalcirudite with reworked bivalves and gastropods as well as scattered pebbles throughout the matrix (facies Bc), 
Table 1 Facies description and sedimentary processes identified along the B17 profile

\begin{tabular}{|c|c|c|}
\hline Facies & Description & Processes \\
\hline Massive mudstone (Mm) & Light gray to dark mudstone without apparent structures & $\begin{array}{l}\text { Deposition of clay and silt under low-energy flow from } \\
\text { suspension }\end{array}$ \\
\hline $\begin{array}{l}\text { Lenticular heterolithic } \\
(\mathrm{Hl})\end{array}$ & $\begin{array}{l}\text { Lenses of greenish gray mudstone connected and/or } \\
\text { isolated interbedded with pale olive calciferous } \\
\text { sandstone. Roots and root marks can be observed on the } \\
\text { top of the facies }\end{array}$ & $\begin{array}{l}\text { Variation of bed load transport by tidal current with } \\
\text { deposition of mud from suspension during very low- } \\
\text { energy periods }\end{array}$ \\
\hline $\begin{array}{l}\text { Biocalcirudite with cross- } \\
\text { stratification }(\mathrm{Bc})\end{array}$ & $\begin{array}{l}\text { Limestone with fossil fragments and quartz pebbles with } \\
\text { cross-stratification and large amounts of bivalves and } \\
\text { gastropods with multi-oriented pattern of deposition }\end{array}$ & $\begin{array}{l}\text { Deposition under moderate- to high-energy flow and } \\
\text { decrease of energy through time. Reworking of fossils } \\
\text { in sand waves with lateral and downstream migration } \\
\text { under bidirectional (with predominance of flood flow) } \\
\text { and combined flows. Fossil and pebbles are left behind, } \\
\text { while sand is transported as bedload during the decrease } \\
\text { of high-energy to moderate-energy flow }\end{array}$ \\
\hline $\begin{array}{l}\text { Massive biocalcirudite } \\
(\mathrm{Bm})\end{array}$ & $\begin{array}{l}\text { Fossiliferous limestone with well-preserved bivalves and } \\
\text { gastropods with multi-oriented pattern of deposition }\end{array}$ & $\begin{array}{l}\text { Sedimentation by high-energy flows, with progressive } \\
\text { decrease through time }\end{array}$ \\
\hline $\begin{array}{l}\text { Sandstone with planar } \\
\text { and/or low-angle cross- } \\
\text { stratification }(\mathrm{Sp})\end{array}$ & $\begin{array}{l}\text { Fine to very fine calciferous sandstone, well-sorted, with } \\
\text { planar and/or low-angle cross-stratification }\end{array}$ & $\begin{array}{l}\text { Deposition of sand under high-energy flow, with } \\
\text { formation of slightly plane beds during swash and } \\
\text { backwash }\end{array}$ \\
\hline Inclined heterolithic (Hi) & $\begin{array}{l}\text { Parallel and inclined beds of light gray mudstone and } \\
\text { sandstone }\end{array}$ & $\begin{array}{l}\text { Lateral accretion with deposition of sand and mud during } \\
\text { low- to moderate-energy flows in a small-scale } \\
\text { meandering channel }\end{array}$ \\
\hline $\begin{array}{l}\text { Calciferous sandstone } \\
\text { (Sca) }\end{array}$ & Fine carbonate sandstone without apparent structures & Deposition of sand under low- to moderate-energy flow \\
\hline
\end{tabular}

and is overlain by inclined sandstone and mudstone (facies $\mathrm{Hi}$ ) and facies $\mathrm{Hl}$. These facies are related to a tidal regime with deposition of mud from suspension and sand from the ebb and flood tide, following the lateral migration of tidal channels with point-bar development (Fig. 3; Table 1). The pollen assemblage of the TF association (Figs. 4, 5, 6) is mainly represented by the mangrove species Zonocostites ramonae (67-76\%), Zonocostites minor (20-24\%) and Deltoidospora adriennis (20-35\%). In general, lowland freshwater forests of Retitricolporites sp. 1 (2-5\%), Retibrevitricolporites (2-5\%) and Mauritiidites franciscoi var. franciscoi (0-3 \%) showed lower percentages, but also presented higher concentrations along the upper tidal flat cycle. Other spores of lowland pteridophytes occur with relatively high concentrations, such as Verrucatosporites sp. 01 (0-20 \%), Polypodiaceoisporites sp. 02 (3-8 \%) and Verrucatosporites usmensis (3-5\%). Foraminifera are very frequent in the lower TF cycle (13-30\%), decreasing in the upper TF cycle (4-10\%), while Ovoidites, a green algae commonly found in freshwater lakes and open-water marsh (Rich et al. 1982), shows an inverse pattern with an increase of concentration values until the upper TF cycle (0-3 to 5-10\%).

Flood-tide delta (FD). The FD association is characterized by biocalcirudites with cross-stratification (facies $\mathrm{Bc}$ ), massive biocalcirudite (facies $\mathrm{Bm}$ ) and conglomerates with pebbles, fragments of bivalves and gastropods in the basal levels that represent channel lag deposits (Fig. 3; Table 1).
This FD association overlies the TF association with an erosive unconformity, likely corresponding to channel dynamics during flood tides and intense rework of sediments from inner shelf/shoreface to lagoon and tidal flat environments under unidirectional and combined flows.

Foreshore $(F S)$. This association is represented by calciferous sandstone with planar or low-angle cross-stratification (facies Sp). Cross-laminations may occur between the main bedding planes. A well-preserved assemblage of crustacean decapods was found in the FS association (Fig. 3; Table 1). This facies is indicative of sediment reworked under oscillatory flows during swash and backwash.

Systematic palynology

Algae

Turma Cystites Potonié and Kremp 1954

Ovoidites Potonié ex Krutzsch 1959

Ovoidites sp. 1

(Fig. 5.1)

Material examined. B17; EF: 25S/4.

Description. Elliptical, psilate ornamentation.

Dimensions. Equatorial length $25.1 \mu \mathrm{m}$, equatorial width $16.5 \mu \mathrm{m}$.

Stratigraphic range. Cretaceous to Recent (Cookson and Dettman 1958). 
Fig. 4 Integrated stratigraphic section, pollen and spore record

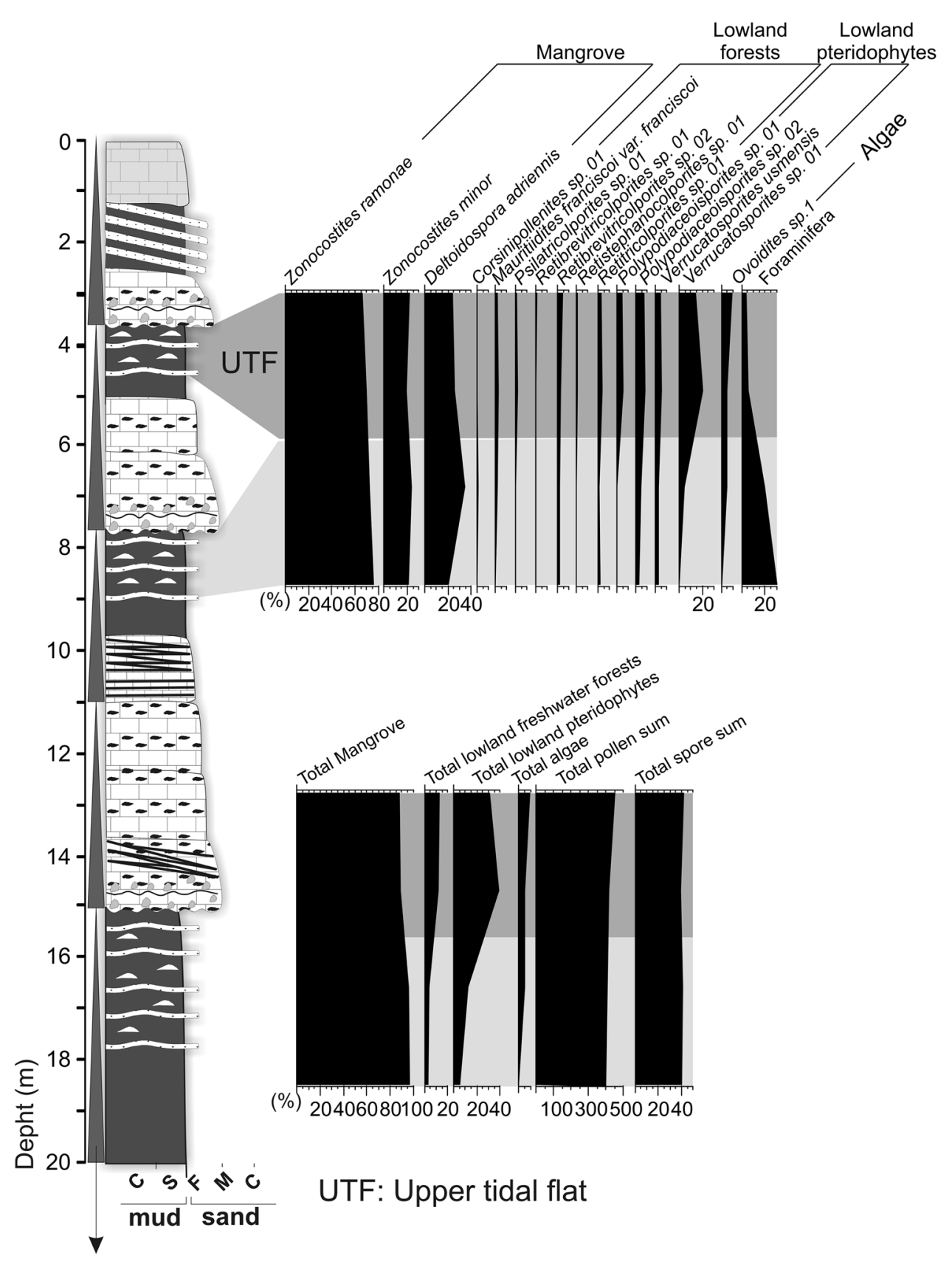

Material examined. B17; EF: 21R/3.

Description. Monolete, bilateral symmetry, elliptical; verrucate ornamentation, verrucae $1.5 \mu \mathrm{m}$ in height and base $1.8 \mu \mathrm{m}$ wide, exine $1 \mu \mathrm{m}$ thick.

Dimensions. Equatorial diameter $52.7 \mu \mathrm{m}$, polar diameter $31.1 \mu \mathrm{m}$.

Stratigraphic range. Upper Eocene-Lower Oligocene to Recent (Lorente 1986; Germerrad et al. 1968). Lower Eocene to Pliocene (Salard-Cheboldaeff 1990), and Upper Eocene to Upper Miocene (Regali et al. 1974).

Germeraad et al. 1968

(Figs. 5.2, 5.3) 

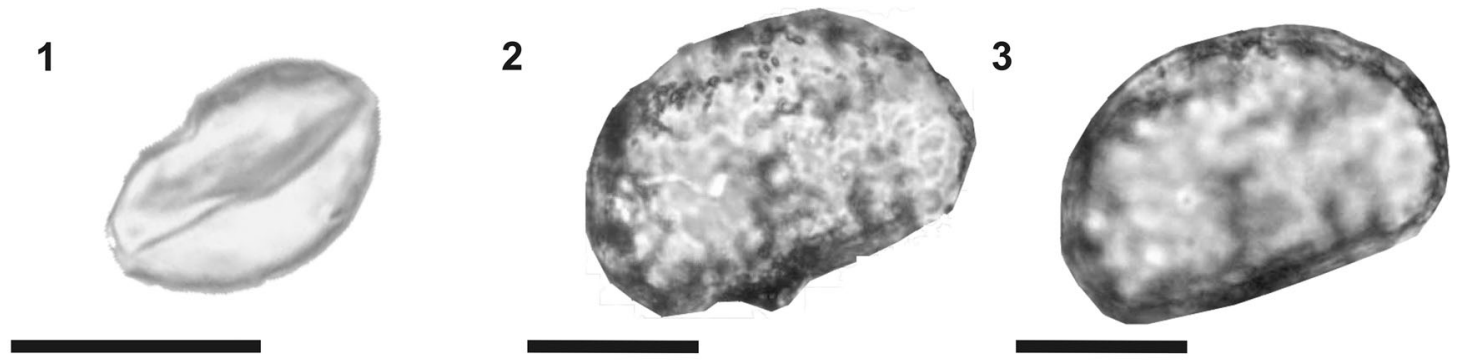

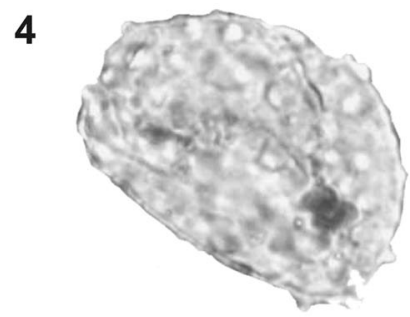

5

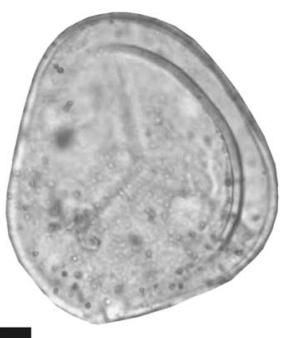

8

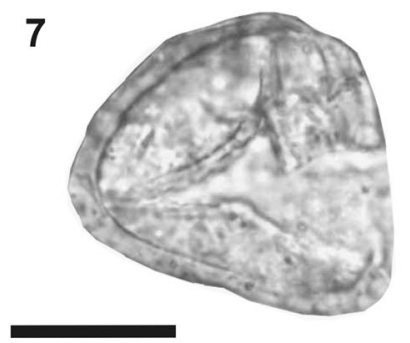

10
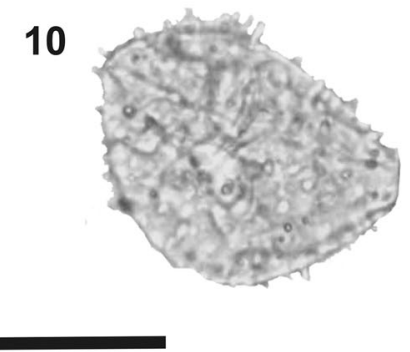

13
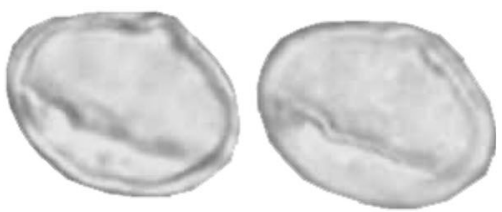

11

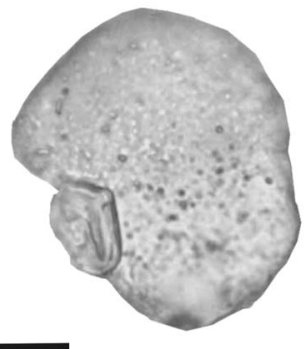

14

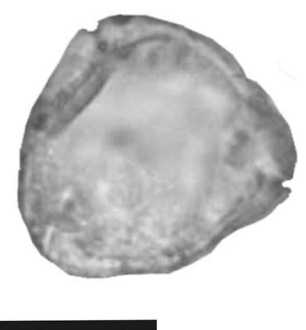

6
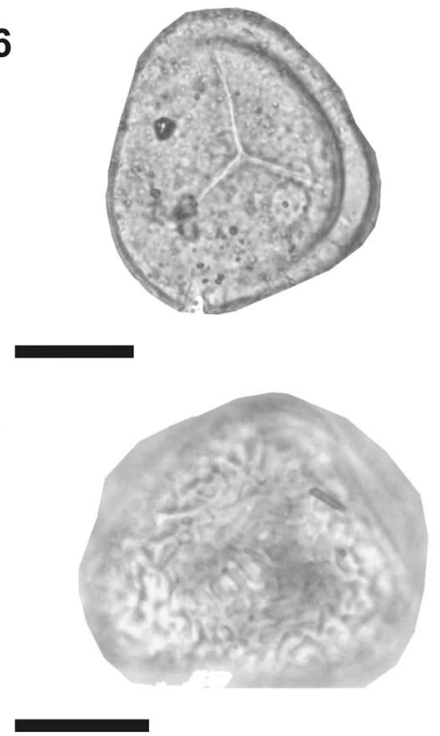

12

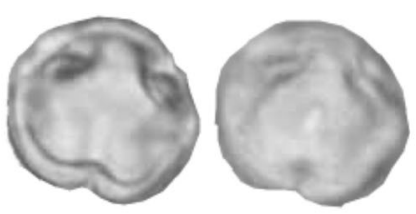

15

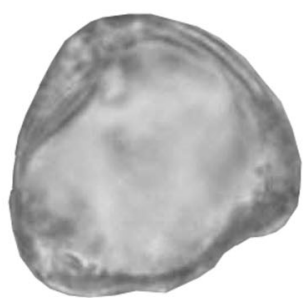

Polypodiaceoisporites sp. 01; 8, Polypodiaceoisporites sp. 02. Distal view, 9, Polypodiaceoisporites sp. 02. Proximal view, 10, Mauritiidites franciscoi var. franciscoi (Van der Hammen 1956a) Van Hoeken-klinkenberg (1964); 11, Corsinipollenites sp. 01; 12, Psilatricolporites sp. 01; 13, Retitricolporites sp. 01; 14, Retibrevitricolporites sp. 01. Proximal view, 15, Retibrevitricolporites sp. 01. Distal view
Fig. 5 Palynological specimens. 1, Ovoidites sp. 01; 2, Verrucatosporites usmensis (Van der Hammen 1956a) Germeraad et al. (1968). Proximal view, 3, Verrucatosporites usmensis (Van der Hammen 1956a) Germeraad et al. (1968). Distal view, 4, Verrucatosporites sp. 01; 5, Deltoidospora adriennis (Potonié and Gelletich 1933) Frederiksen et al. (1983). Distal view, 6, Deltoidospora adriennis (Potonié and Gelletich 1933) Frederiksen et al. (1983). Proximal view, 7 , 
Fig. 6 Palynological specimens. 1 , Retibrevitricolporites sp. 02

Retibrevitricolporites sp. 02. Distal view; 3, Zonocostites ramonae Gemeraad et al., 1968. Type 1; 4, Zonocostites ramonae Gemeraad et al., 1968. Type 2; 5, Zonocostites minor Jaramillo and Dilcher, 2001. Distal view; 6, Zonocostites minor Jaramillo and Dilcher, 2001. Proximal view; 7 , Retistephanocolporites sp. 01. Distal view; 8 ,

Retistephanocolporites sp. 01. Proximal view Proximal view; 2 ,
1

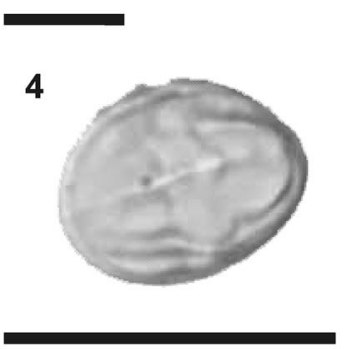

7

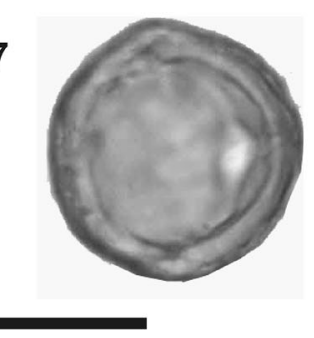

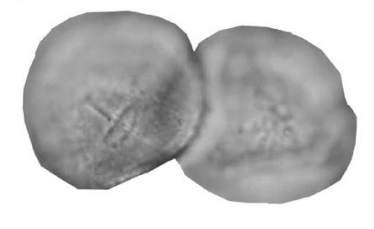

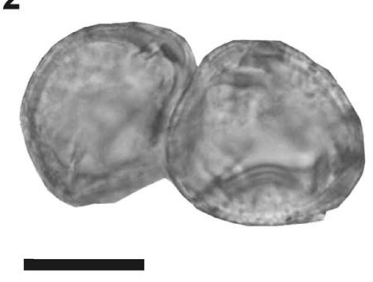

5

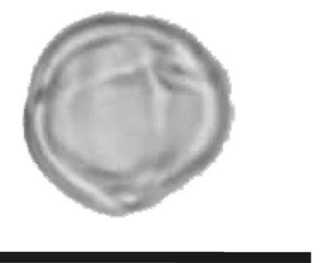

3

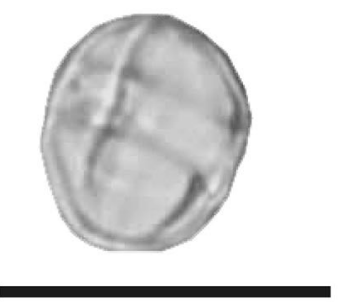

6

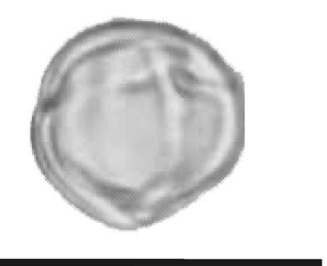

Taxonomic affinity. The species resembles Stenochlaena palustris of Blechnaceae (Germeraad et al. 1968). Polypodim-Polypodiaceae (Lorente 1986).

Comparisons. Laevigatosporites ovatus Wilson and Webster 1946 has thinner exine (e.g., Santos et al. 2005).

\section{Verrucatosporites $\mathrm{sp} .01$}

(Fig. 5.4)

\section{Material examined. B17; EF: 34W.}

Description. Monolete, bilateral symmetry, elliptical; verrucate ornamentation, verrucae $1.5 \mu \mathrm{m}$ in height and base $2.1 \mu \mathrm{m}$ wide, exine $0.8 \mu \mathrm{m}$ thick.

Dimensions. Equatorial diameter $39.4 \mu \mathrm{m}$, polar diameter $27.6 \mu \mathrm{m}$.

Taxonomic affinity. Polypodim-Polypodiaceae.

Comparisons. Polypodiisporites specious Sah 1967 and Verrucatosporites specious Harris, 1965 have wider verrucae $(4-7 \mu \mathrm{m})$ and thicker intexine $(1.5-2 \mu \mathrm{m})$.

Triletes Reinsch emend. Dettmann, 1963

Deltoidospora Miner 1935

Deltoidospora adriennis (Potonié and Gelletich 1933) Frederiksen 1983

(Figs. 5.5. 5.6)

Material examined. B17; EF: 11R.

Description. Trilete, anisopolar, radially symmetric, triangular-obtuse-convex, curvature absent, margo absent, commissure straight, exine $1.5 \mu \mathrm{m}$ thick, psilate to micropitted ornamentation.

Dimensions. Equatorial length $50.6 \mu \mathrm{m}$, equatorial width $46.5 \mu \mathrm{m}$.

Stratigraphic range. Upper Eocene (Santos et al. 2010), Lower Oligocene to Recent (Lorente 1986).

Taxonomic affinity. Acrostichum auerum L. (Hoorn 1993).

Comparisons. Camarozonosporites sp. 1 Jaramillo and Dilcher 2001 has a rugulate distal face and is smaller in size $(27-30 \mu \mathrm{m})$. Matonisporites mulleri Playford 1982 has curvatura perfecta and psilate ornamentation over the entire spore grain. Deltoidospora juncta (Kara Murza 1956) Singh 1964 is smaller (32-34 $\mu$ m diameter).

Polypodiaceoisporites Potonié 1951 ex Potonié 1956 Polypodiaceoisporites sp. 01

(Fig. 5.7)

Description. Trilete, radially symmetric, triangularobtuse-convex, curvatura perfecta; radii long, reaching the equator without extending into cingulum; cingulum psilate, $2.9 \mu \mathrm{m}$ thick; reticulate ornamentation, lumina $1 \mu \mathrm{m}$ wide and muri $0.9 \mu \mathrm{m}$ wide.

Dimensions. Equatorial length $41.5 \mu \mathrm{m}$, equatorial width $38.5 \mu \mathrm{m}$.
Material examined. B17; EF: 17T/2. 
Taxonomic affinity. Polypodiaceae.

Comparisons. Polypodiaceoisporites fossulatus Jaramillo and Dilcher (2001) has the equatorial outline, which is triangular-obtuse-straight and fossulate ornamentation. Polypodiaceoisporites pseudopsilatus Lorente 1986 is foveolate on the distal face and psilate on the proximal face.

Polypodiaceoisporites sp. 02

(Figs. 5.8, 5.9)

Material examined. B17; 44Q.

Description. Trilete, radially symmetric, triangular-obtuse, curvatura absent; radii long, reaching the equator without extending into cingulum, labrum $2.5-3 \mu \mathrm{m}$ wide; cingulum reticulate, interradial thickening, cingulum $3.8 \mu \mathrm{m}$ thick, radial thinning, cingulum $<0.5 \mu \mathrm{m}$ thick; rugulate ornamentation, muri 1-2 $\mu \mathrm{m}$ wide, lumina $1-2 \mu \mathrm{m}$ wide.

Dimensions. Equatorial length $52 \mu \mathrm{m}$.

Taxonomic affinity. Polypodiaceae.

Comparisons. Polypodiaceoisporites pseudopsilatus Lorente 1986 has a cingulum of 2-3 $\mu \mathrm{m}$ thickness, a thinner labrum ( $2 \mu \mathrm{m}$ wide) and a psilate cingulum. Psilatriletes lobatus Hoorn 1994b has a psilate ornamentation and a wider cingulum at the interradial region.

Angiosperms

Monocolpatae Iversen and Troels-Smith 1950

Mauritiidites Van Hoeken-Klinkenberg 1964

Mauritiidites franciscoi var. franciscoi (Van der Hammen 1956a) Van Hoeken-Klinkenberg 1964

(Fig. 5.10)

Material examined. B17; 27N/2.

Description. Monad, anisopolar, elliptical, monocolpate, monosulcate, sulcus simple intectate, exine 1-2 $\mu \mathrm{m}$ thick; echinate ornamentation, spines 2-4 $\mu \mathrm{m}$ height and 1-2 $\mu \mathrm{m}$ wide, conical.

Dimensions. Equatorial length $41.5 \mu \mathrm{m}$, equatorial width $34.5 \mu \mathrm{m}$.

Stratigraphic range. Paleocene to Pleistocene (Muller et al. 1987).

Taxonomic affinity. Mauritia-Arecaceae (Hoorn 1993).

Comparisons. Mauritiidites franciscoi var. minutes Van der Hammen and Garcia 1966 is smaller and also has shorter spines $(0.5-1 \mu \mathrm{m}$ height).

Triporatae Iversen and Troels-Smith 1950

Corsinipollenites Nakoman 1965

Corsinipollenites sp. 01

(Fig. 5.11)
Material examined. B17; EF: 17G.

Description. Monad, radially symmetric, anisopolar, elliptical; triporate, ecto and endospore coinciding, pore circular, $4.6 \mu \mathrm{m}$ diameter; costate, costae $2 \mu \mathrm{m}$ wide, annulus 4-5 $\mu \mathrm{m}$ wide; scabrate ornamentation, exine $1.5 \mu \mathrm{m}$ thick and it thickens to 2.5-3 toward the pores.

Dimensions. Equatorial length $44 \mu \mathrm{m}$, equatorial width $38 \mu \mathrm{m}$.

Taxonomic affinity. Onagraceae-Ludwigia.

Comparisons. Corsinipollenites scabratus Silva-Caminha et al. 2010 has a distinct Y mark in one of the poles. Corsinipollenites psilatus Jaramillo and Dilcher 2001 is psilate. Corsinipollenites oculusnoctis (Thiegart 1940) Nakoman 1965 has also a Y mark and a thinner annulus (2-3 $\mu \mathrm{m})$.

Tricolporatae Iversen and Troels-Smith 1950

Psilatricolporites (Van der Hammen 1956a) Van der Hammen and Wymstra 1964

Psilatricolporites sp. 01

(Fig. 5.12)

Material examined. B17; EF: 35Q/4.

Description. Monad, radially symmetric, isopolar, elliptical, tricolporate, distinct colpi and pores; costate, costae $1 \mu \mathrm{m}$ thick, exine $1.4 \mu \mathrm{m}$ thick.

Dimensions. Equatorial length $11.3 \mu \mathrm{m}$, equatorial width $10.4 \mu \mathrm{m}$.

Taxonomic affinity. Caesalpiniodeae.

Comparisons. Psilatricolporites costatus Dueñas 1980 has a distinct columella and a larger equatorial length (18-24 $\mu \mathrm{m})$.

Retitricolporites Van der Hammen and Wymstra 1964.

Retitricolporites sp. 01

(Fig. 5.13)

Material examined. B17; EF: 22Q.

Description. Monad, radially symmetric, isopolar, elliptical, tricolporate, distinct colpi, ectocolpi costate, reticulate ornamentation, lumina $0.4 \mu \mathrm{m}$, muri $0.3 \mu \mathrm{m}$ wide, exine $1 \mu \mathrm{m}$ thick.

Dimensions. Polar length $15.3 \mu \mathrm{m}$, polar width $11.5 \mu \mathrm{m}$.

Taxonomic affinity. Papilionoideae.

Comparisons. Striatricolporites poloreticulatus SilvaCaminha et al. 2010 has a striate to reticulate ornamentation and a larger size (23 to $28 \mu \mathrm{m})$. 
Retibrevitricolporites Legoux 1978

Retibrevitricolporites sp. 01

(Figs. 5.14, 5.15)

Material examined. B17; EF: 21T/1.

Description. Monad, radially symmetric, isopolar, triangular-obtuse-convex, tricolporate, colpi short, exine thickens toward the colpi and pore margins, costate, costae $2 \mu \mathrm{m}$ thick; nexine $1.2 \mu \mathrm{m}$ thick, sexine $1 \mu \mathrm{m}$, reticulate ornamentation, lumina $0.8 \mu \mathrm{m}$ wide, muri $0.7 \mu \mathrm{m}$ wide.

Dimensions. Equatorial length $27.2 \mu \mathrm{m}$, equatorial width $25 \mu \mathrm{m}$.

Taxonomic affinity. Papilionoideae.

Comparisons. Syncolporites poricostatus Van HoekenKlinkenberg 1966 is parasyncolporate and has a smaller equatorial length and width (16 and $12 \mu \mathrm{m}$, respectively). Retibrevitricolporites triangulatus Van Hoeken-Klinkenberg 1966 has a larger lumina and muri $(1.5 \mu \mathrm{m}$ wide $)$.

Retibrevitricolporites sp. 02

(Figs. 6.1, 6.2)

Material examined. B17; 28G.

Description. Monad, radially symmetric, isopolar, elliptical, tricolporate, colpi short, distinct colpi and pores; ectocolpi costate, costae $3 \mu \mathrm{m}$ thick, distinct; nexine $1.3 \mu \mathrm{m}$ thick, sexine $1.6 \mu \mathrm{m}$ thick, elliptical pore with $7 \times 3 \mu \mathrm{m}$; collumelate, collumelae $0.9 \mu \mathrm{m}$; reticulate ornamentation, lumina $0.8 \mu \mathrm{m}$ wide, muri $0.7 \mu \mathrm{m}$ wide.

Dimensions. Equatorial length $36 \mu \mathrm{m}$, equatorial width $31 \mu \mathrm{m}$.

Taxonomic affinity. Caesalpinioideae.

Comparisons. Retibrevitricolporites yavarensis (Hoorn 1993) Silva-Caminha et al. 2010 has a smaller equatorial length and width $(20 \mu \mathrm{m})$, lumina and muri $(0.5 \mu \mathrm{m}$ wide $)$.

Zonocostites Gemeraad et al. 1968

Zonocostites ramonae Gemeraad et al. 1968

(Figs. 6.3, 6.4)

Material examined. B17; EF: 14Y/1.

Description. Monad, radially symmetric, isopolar, elliptical in equatorial view, tricolporate, distinct pores and colpi, zonorate, costate, costae $1,5 \mu \mathrm{m}$ thick, pore $1,8 \mu \mathrm{m}$ diameter, reticulate ornamentation, muri $0,8 \mu \mathrm{m}$ wide, lumina $1 \mu \mathrm{m}$ wide, exine $1,8 \mu \mathrm{m}$ thick.

Stratigraphic range. Lower-Upper Eocene to Recent (Gemeraad et al. 1968; Muller et al. 1987).

Dimensions. Polar length $28 \mu \mathrm{m}$, polar width $25 \mu \mathrm{m}$.
Taxonomic affinity. Rhizophora mangle $\mathrm{L}$.

Comparisons. Zonocostites minor (Jaramillo and Dilcher 2001) has smaller dimensions and is micropitted. Zonocostites duquei Dueñas 1980 has distinct collumelae.

Zonocostites minor Jaramillo and Dilcher 2001

(Figs. 6.5, 6.6)

Material examined. B17; EF: 14 W/1.

Description. Monad, radially symmetric, isopolar, elliptical in equatorial view, tricolporate, distinct pores and colpi, pore diameter $0.5-0.6 \mu \mathrm{m}$, zonorate, costate, costae $0.5-0.7 \mu \mathrm{m}$ thick, exine $0.8-1 \mu \mathrm{m}$ thick, slightly reticulate to micropitted ornamentation.

Stratigraphic range. Lower-Upper Eocene to Recent (Gemeraad et al. 1968; Muller et al. 1987).

Dimensions. Equatorial diameter 12-14 $\mu \mathrm{m}$, polar diameter $11-12 \mu \mathrm{m}$.

Taxonomic affinity. Mangrove.

Comparisons. Zonocostites ramonae Gemeraad et al. 1968 has similar features but is of larger size $(26-19 \mu \mathrm{m})$ and has a reticulate ornamentation.

Retistephanocolporites Van der Hammen and Wymstra 1964

Stephanocolporate Iversen and Troels-Smith 1950

Retistephanocolporites sp. 01

(Figs. 6.7, 6.8)

Material examined. B17; EF: 27K.

Description. Monad, radially symmetric, isopolar, circular, stephanocolporate (6 colpi and pores), distinct pores and colpi, colpi short, costate, costae $1.4 \mu \mathrm{m}$ thick, pore $3.2 \mu \mathrm{m}$ diameter; exine $4 \mu \mathrm{m}$ thick; slightly reticulate ornamentation.

Dimensions. Total diameter $28 \mu \mathrm{m}$.

Taxonomic affinity. Diacidia-Malpighiaceae.

Systematic paleontology

Crustacea

Decapoda Latreille 1802

Callianassoidae Dana 1852

Neocallichirus Sakai 1988

Neocallichirus sp.

(Fig. 7.1)

Material examined. MPEG-2340-I (4/4). Propodi.

Occurrence. The specimens were collected from the Early Miocene Pirabas Formation, Capanema Mine B17 $\left(1^{\circ} 10^{\prime} \mathrm{S}\right.$, $\left.47^{\circ} 13^{\prime} \mathrm{W}\right)$. 


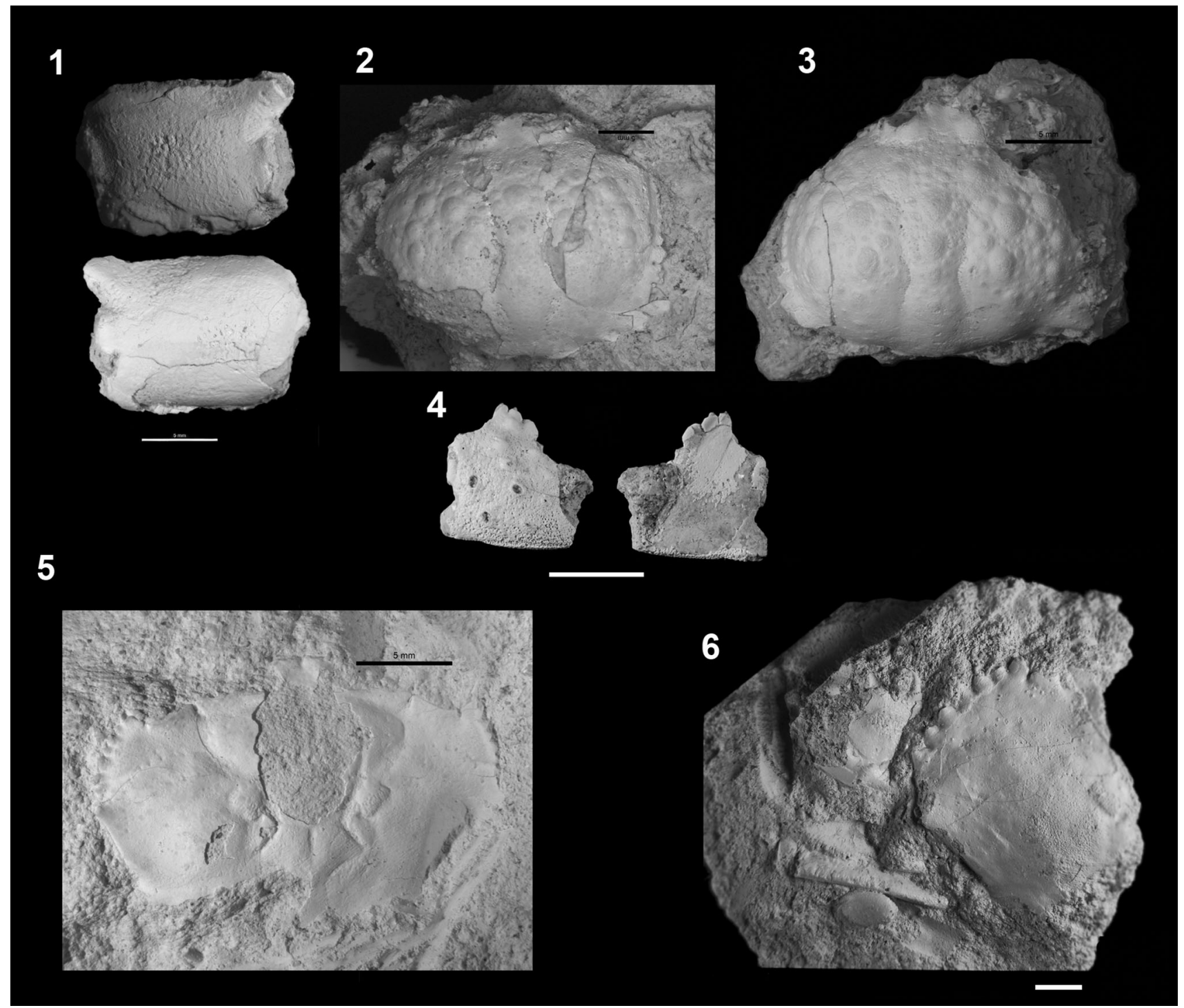

Fig. 7 Crustacean Decapods. 1 Neocallichirus sp. MPEG-2340-I, propodi, outer and inner view; 2,3 Calappila circularis, MPEG-2337I, MPEG-2338-I, carapaces, dorsal view; 4 Calappila sp. MPEG-

Description. Manus rectangular, slightly highest proximally, both margins slightly serrated, bulbous, swelling; fixed finger stronger and broken, proximal section gently curved dorsally, movable finger unpreserved.

Calappidae Milne Edwards 1873

Calappila Milne Edwards 1873

Calappila circularis (Beurlen 1958) Martins-Neto 2001

(Figs. 7.2-7.3)

Material examined. MPEG-2337-I, MPEG-2338-I. Carapace.

Occurrence. The specimens were collected from the Early Miocene Pirabas Formation, Capanema Mine B17 $\left(1^{\circ} 10^{\prime} \mathrm{S}\right.$, $\left.47^{\circ} 13^{\prime} \mathrm{W}\right)$
2339-I, chela, outer and inner view; 5 Euphylax septendentatus, MPEG-2333-I, carapace, dorsal view; 6 Necronectes sp., MPEG2331-I, carapace, dorsal view

Description. Carapace ovoid, widest at about midlength; anterolateral margin smooth, lacking prominent lateral spine, posterolateral margin with progressively welldeveloped spines developed on flares extension; surface coarsely nodose; nodes and grooves define carapace regions.

\section{Calappila sp.}

(Fig. 7.4)

Material examined. MPEG-2339-I. Left chela.

Occurrence. The specimen was collected from the Early Miocene Pirabas Formation, Capanema Mine B17 $\left(1^{\circ} 10^{\prime} \mathrm{S}\right.$, $\left.47^{\circ} 13^{\prime} \mathrm{W}\right)$. 
Description. Palm highest distally with almost three preserved spines and one broken in the dorsal border; external surface coarsely nodose; movable finger unpreserved.

Portunidae Rafinesque 1815

Euphylax Stimpson 1860

Euphylax septendentatus Beurlen 1958

(Fig. 7.5)

\section{Material examined. MPEG-2333-I, MPEG-2334-I.} Carapace.

Occurrence. The specimen was collected from the Early Miocene Pirabas Formation, Capanema Mine B17 $\left(1^{\circ} 10^{\prime} \mathrm{S}\right.$, $\left.47^{\circ} 13^{\prime} \mathrm{W}\right)$.

Description. Carapace hexagonal, wider than long, length about two-thirds the maximum width, widest at about half the distance posteriorly; front T-shaped, often with sharp central spine; fronto-orbital width about $80 \%$ maximum carapace width; orbit with granular rim, sometimes with two closed fissures positioned near the distal end, sometimes with concave reentrant near the outer-orbital angle to embrace eye; anterolateral margin usually with three to five spines including outer-orbital spine; transverse ridge extending from last anterolateral spine axially on dorsal carapace; transverse ridges often on protogastric and hepatic regions.

Necronectes Milne Edwards 1881

Necronectes sp.

(Fig. 7.6)

Material examined. MPEG-2331-I, MPEG-2332-I. Carapace.

Occurrence. The specimen was collected from the Early Miocene Pirabas Formation, Capanema Mine B17 ( $1^{\circ} 10^{\prime} \mathrm{S}$, $\left.47^{\circ} 13^{\prime} \mathrm{W}\right)$.

Description. Carapace wider than long; axial regions generally well developed; dorsal surface covered with very fine granules; protogastric region defined by two circular swellings; mesogastric region broad posteriorly, narrowing anteriorly, well defined by grooves posteriorly and laterally, poorly defined anteriorly; cardiac region circular, elevated. Frontal margin with six spines including innerorbital spine; spines small, blunt tipped. Anterolateral margin generally longer than posterolateral margin; eight spines on anterolateral margin including outer-orbital spine; spines increasing in size posteriorly except for eighth spine which is generally smaller than adjacent spine; spines triangular, with pointed tips; moving posteriorly spines become increasingly curved anteriorly. The ventral surface from the examined specimen is included in the matrix.
Portunus Weber 1795

Portunus haitiensis Rathbun 1924

(Figs. 8.1, 8.5)

Material examined. MPEG-2323-I to MPEG-2328-I, MPEG 2352-I, MPEG 2354-I. Carapace.

Occurrence. The specimens were collected from the Early Miocene Pirabas Formation, Capanema Mine B17 $\left(1^{\circ} 10^{\prime} \mathrm{S}\right.$, $47^{\circ} 13^{\prime} \mathrm{W}$.

Description. Carapace much wider than long; carapace regions moderately developed; frontal and anterolateral spines are unpreserved in all specimens collected; abdomen enlarged, with somites 3-5 fused; the second abdominal is pentagonal.

Portunus pirabensis Martins-Neto 2001

(Figs. 8.6, 8.7)

Material examined. MPEG-2329-I, MPEG-2330-I, MPEG 2353-I. Carapace.

Occurrence. The specimens were collected from the Early Miocene Pirabas Formation, Capanema Mine B17 $\left(1^{\circ} 10^{\prime} \mathrm{S}\right.$, $\left.47^{\circ} 13^{\prime} \mathrm{W}\right)$.

Description. Carapace much wider than long; carapace regions moderately developed; frontal and anterolateral spines are unpreserved in all specimens collected; abdomen enlarged, with somites $3-5$ fused; the second abdominal is trapezoidal, with the posterior margin circa one and half times longer that the anterior margin.

Scylla Haan 1833

Scylla aff. costata (Rathbun 1924) Beurlen 1958

(Fig. 8.8)

Material examined. MPEG-2351-I. Carapace.

Occurrence. The specimen was collected from the Early Miocene Pirabas Formation, Capanema Mine B17 $\left(1^{\circ} 10^{\prime} \mathrm{S}\right.$, $47^{\circ} 13^{\prime} \mathrm{W}$.

Description. Carapace about twice as wide as long, ovate, maximum width just over half the distance posteriorly; regions poorly defined, carapace smooth; front spines unpreserved in the examined specimen; anterolateral margins with nine spines including outer-orbital spines, last spine longest; gastric grooves strong. The ventral surface from the examined specimen is included in the matrix.

Portunidae, genera and species indeterminate

(Figs. 8.9, 8.12)

Material examined. MPEG-2341-I to MPEG-2350-I. Chela.

Occurrence. The specimens were collected from the Early Miocene Pirabas Formation, Capanema Mine B17 $\left(1^{\circ} 10^{\prime} \mathrm{S}, 47^{\circ} 13^{\prime} \mathrm{W}\right)$. 


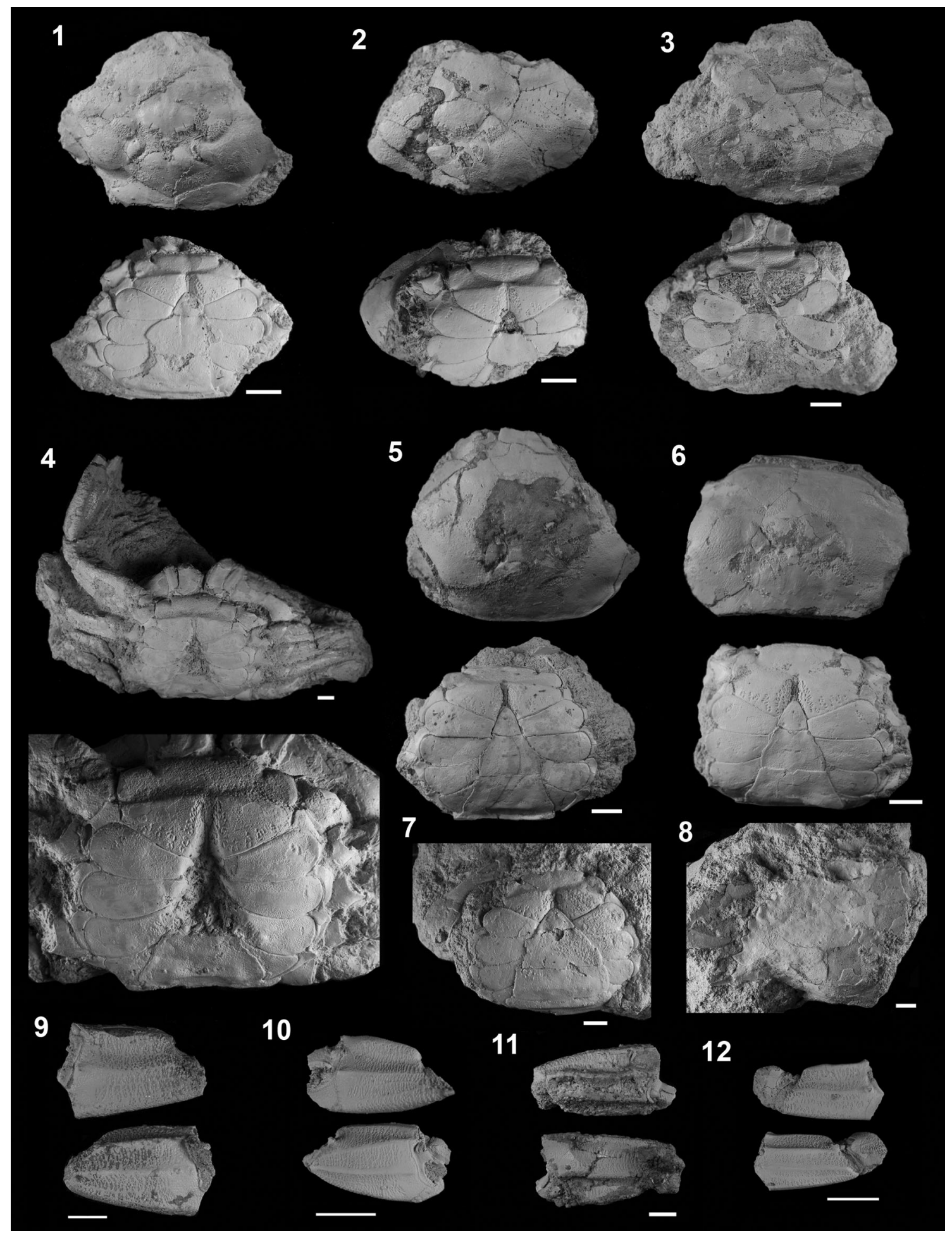

Fig. 8 Crustaceans Decapods. 1-5, Portunus haitiensis, MPEG2323-I to MPEG-2327-I, carapace, dorsal and ventral view (except 4 only ventral view and enlarge details); 6, 7, Portunus pirabensis,
MPEG-2329-I, MPEG-2330-I, carapace, ventral view; 8, Scylla aff. costata, MPEG-2351-I, carapace, dorsal view; 9-12, Portunidae, chelas, outer and inner surface. Scale bar $10 \mathrm{~mm}$ 
Description. Chelipeds massive, with very weak keels on outer surface palm, sometimes with two distal spines on upper margin; surface with anastomosed reticulations.

Type species of Crustacean Decapods from the Pirabas Formation, Pará state, Brazil.

$\dagger$ Calappilia circularis Beurlen 1958. MN-4619-I. Loc. Salinópolis e Ponta da Fazenda. (Schweitzer et al. 2009: 23 extinct species are included in this genus).

$†$ Sesarma paraensis Beurlen 1958. DNPM? (Without catalog data). Loc. Colônia Pedro Teixeira (Schweitzer et al. 2009: 2 extinct species).

$\dagger$ Parthenope trituberculata Beurlen 1958. DNPM-4459. Loc. Colônia Pedro Teixeira (Schweitzer et al. 2009: 13 extinct species).

$\dagger$ Callinectes paraensis Beurlen 1958. MN-4585-I. Loc. Colônia Pedro Teixeira (Schweitzer et al. 2009: 8 extinct species).

$\dagger$ Euphylax septendentatus Beurlen 1958. DNPM-4457. Loc. Rio do peixe Boi, Capanema (Schweitzer et al. 2009: 6 extinct species).

$\dagger$ Hepatella amazonica Beurlen 1958. DNPM-4458. Loc. Colônia Pedro Teixeira (Schweitzer et al. 2009: monospecific).

$\dagger$ Cyclocancer tuberculata Beurlen 1958. MN-4617-I. Loc. Colônia Pedro Teixeira e ilha de Fortaleza (Schweitzer et al. 2009: monospecific).

$\dagger$ Typilobus unispinatus Martins-Neto 2001. DNPM?. Without catalog data. Loc. Ponta da Fazenda (Schweitzer et al. 2009: 17 extinct species and 10 species are dubious). $\dagger$ Portunus pirabaensis Martins-Neto 2001. MN-5402, MN5400, MN5401. Loc. Colônia Pedro Teixeira (Schweitzer et al. 2009: 48 extinct species).

$\dagger$ Paratumidocarcinus marajoarus Martins-Neto 2001. RGMN-T06. Loc. Colônia Pedro Teixeira. (Schweitzer et al. 2009: monospecific).

$\dagger$ Panopeus capanemaensis Martins-Neto 2001. MN-5012-

I. Loc. Rio Japerica, Primavera (Schweitzer et al. 2009: 22 extinct species).

$\dagger$ Uca inaciobritoi Martins-Neto 2001. MN-5014-I, MN5015-I, UFRJ- IG-242, 264, 265. Loc. Furo de Baunilha Grande (Schweitzer et al. 2009: 10 extinct species).

\section{Discussion}

Faunal assemblage interpretation

Callianassidae are burrowers, constructing lengthy domicile galleries in nearshore or intertidal sediments (Manning and Felder 1991; Aguilera et al. 2010). Neocallichirus, common in the Mine B17, inhabited a paleoenvironment characterized by shallow waters, soft bottom and sandy shorelines in intertidal or shallow subtidal rear-shore habitats. The most abundant bioturbation causes in the field are those generated by burrows of Neocallichirus.

$\dagger$ Calappilia (an extinct, speciose genus within the Calappidae) and its inferred paleoenvironment is based on comparison with the extant genera Calappa and interpreted as shallow water over coral reefs, shelly, sandy or muddy substrate (Bellwood 1998; Aguilera et al. 2010).

Portunidae are swimmers and back-burrow crabs. This 'family' forms a monophyletic group, and their first occurrence in the Eocene is followed by major Miocene radiation (Karasawa et al. 2008). The occurrence of portunids in the stratigraphic section of the Capanema Mine B17 includes Euphylax, †Necronectes, Portunus and Scylla, whose ecology is in agreement with the facies interpretation treated here and other fossil portunid records in the tropical Americas (Aguilera et al. 2010). These genera are associated with shallow waters and neritic coastal paleoenvironments over sandy and soft bottom, beaches, estuaries, and in marginal lagoons with sea grass and mangrove protected area.

Euphylax is known primarily from the Miocene, when the genus was apparently most speciose in the Caribbean and Central America (Nyborg et al. 2003; Schweitzer et al. 2006). Fossil species of Necronectes (an extinct genus within the Portuninae) are known from the Oligocene and Miocene along the east coast of North America, Central America, Caribbean islands, South America and from Tethyan Europe (Schweitzer et al. 2006). Scylla appeared during the late Oligocene or early Miocene and its occurrences in circumtropical and equatorial regions during Miocene is a relict of a once broader distribution (Schweitzer et al. 2006).

The status of several other specimens of Portunidae remains that of unidentified species, because they are based solely on fragmented carapaces or isolated chelipeds.

\section{Paleoenvironmental interpretation}

Aguilera and Páes (2012) distinguished the Capanema B17 Mine faunal assemblage from other four outcrops of the Pirabas Formation in the vicinity (Ilha de Fortaleza, Estação Agronômica, Aricuru, and Colônia Pedro Teixeira) based on the PCA analysis. Their observation is corroborated by the facies analysis previously developed by Petri (1957) as ecofacie Caneco (=Capanema) for "littoral coastal marine to transitional basin border, reaching $10 \mathrm{~m}$ deep", a frequently cited facies name in literature (e.g., Távora et al. 2007). The sedimentary system in the Capanema Mine B17 section treated here is interpreted as a transitional tidal platform with infaunal and epifaunal invertebrate richness associated with a high calcareous 
algal productivity (photic zone), as indicated by the presence of portunids (blue crabs) and Calappidae (box crabs) and their preference for shallow water littoral to tidal influence and marginal coastal lagoons under brackish paleoenvironment.

The lagoonal strata along the section studied show a thickness similar to deposits of the tidal flat with mangrove vegetation. The mangrove vegetation, with strong marine influence as indicated by high amounts of foraminifera, remained relatively stable in the tidal flats area over time during the evolution of this environment. However, the increase of species diversity from freshwater forests, lowland pteridophytes and freshwater algae, coupled with decrease in foraminifera diversity in the upper tidal flat deposit is interpreted as an indication of a slight increase of precipitation rates and freshwater inflow.

Divergent paleoenvironmental conclusions were brought forward by previous researchers as open continental shelf with shallow tropical water, moderate- to high-energy flow and a wave-dominated estuarine environment (Góes et al. 1990; Rossetti and Góes 2004) and extensive lagoon system (Távora et al. 2007). However, the successions suggest more heterogeneity of laterally coexisting environments in a coastal depositional system (Walker 1992) and attempts to honor all faunal, floral and sedimentological data observed. The majority of Oligocene-Miocene Caribbean decapods suggest that they preferred clastic bottom, with a variety of settings, including sea grass mud plains, calcareous detrital environments, siliciclastic ramps, deltas and quiet lagoons (Schweitzer et al. 2006).

\section{Paleogeographic interpretation}

During the early Miocene, extensive carbonate platforms were present along the continental margin in the eastern Amazon, northern Brazil, represented in the Pirabas Formation, characterized by diverse and abundant marine fauna, closely related to similar assemblages known from the Oligocene-Miocene carbonate deposit of the Caribbean

When the Andes cordillera progressively uplifted from the late Oligocene through the late Miocene, the modern South American hydrographic basins were established, and the Amazon River began shifting its drainage and sediment input to the Atlantic coast (e.g., Hoorn et al. 2010). This resulted in a drastic reduction of carbonate deposition and establishment of clastic dominated environments influenced by the continuous water currents from the eastern Amazon and eastern Caribbean along the marine coastal environments where the Amazon-Orinoco deltas discharged freshwater flow and great quantities of siliclastics into the sea. The geological and oceanographic barrier, established since the late early Miocene until today, results in marine biota of the area to be poorly connected with Caribbean biota proper. Based on the observed fossil record, the Neogene tropical western central Atlantic paleobiogeographic sub-province was erected (Aguilera et al. 2011; Aguilera and Páes 2012) and the Brazilian biogeographic province was established separately based on extant faunal distribution (e.g., Pérez-Ruzafa et al. 2012).

\section{Conclusion}

The integration of facies, pollen and spores, and crustacean decapod data led to the identification of a carbonate/siliciclastic transitional depositional sedimentary system with lagoon, tidal flat, flood-tidal delta and foreshore environments. The tidal flats were predominantly colonized by mangrove species such as Zonocostites ramonae, Zonocostites minor and Deltoidospora adriennis. Crustacean decapods of Euphylax, $\uparrow$ Necronectes, Portunus and Scylla genera were abundant in the foreshore environment, and are also indicative of the presence of shallow water and marginal lagoons with mangrove protected areas. The formation of these extensive carbonate environments is thought to be related to the lack of large continental drainage systems such as the Amazon River basin.

Acknowledgments This research was supported by the Museum Paraense Emilio Goeldi, the Conselho Nacional de Desenvolvimento Científico e Tecnológico (Process CNPq 311783/2011-2 to OA and CNPq 565046/2010-1 to HM-S), the Instituto Tecnológico Vale Desenvolvimento Sustentável and the Instituto de Geociencias-Universidade Federal do Pará in Brazil. Peter Toledo, Sue Costa and Maria Ines Feijó participated during the geological survey in the Capanema Mine B17 and helped to collect fossil crabs during field trips. We thank Zoneibe Luz for support in the preparation of the fossil crabs and photographs. The senior author thanks Maria de Lourdes Ruivo for support in the PV-MPEG-CNPq project (310009/2011-2). We thank Francisco Vega and Vladimir Távora for bibliographic support, comments and suggestions. We thank the anonymous reviewers and editors for their corrections and suggestions. Special thanks are due to Werner Schwarzhans (Hamburg, Germany) for further improvement of the manuscript.

\section{References}

Aguilera, O., \& Páes, E. (2012). The Pirabas Formation (Early Miocene from Brazil) and the Tropical Western Central Atlantic subprovince. Boletim do Museu Paraense Emilio Goeldi Ciências Naturais, 7(1), 29-45.

Aguilera, O., Ramos, M. I., Páes, E., Costa, S., \& Sánchez-Villagra, M. (2011). The Neogene tropical American fish assemblage and the paleobiogeography of the Caribbean region. Swiss Journal of Palaeontology, 130, 1-24.

Aguilera, O., Rodrigues de Aguilera, D., Vega, F. J., \& SánchezVillagra, M. (2010). Mesozoic and Cenozoic decapod crustaceans from Venezuela and related trace-fossil assemblages. In 
M. Sánchez-Villagra, O. Aguilera, \& F. Carlini (Eds.), Urumaco and Venezuelan paleontology (pp. 103-128). Bloomington: Indiana University Press.

Arai, M. (1997). Dinoflagelados (Dynopniceae) miocênicos do Grupo Barreiras do nordeste do Estado do Pará (Brasil). Revista da Universidade de Guarulhos, 2, 98-106.

Beardsley, R., Candela, J., Limeburner, R., Gleyer, W., Lentz, S. J., Castro, B. M., et al. (1995). The M1 tide on the Amazon shelf. Journal of Geophysical Research, 100, 2283-2319.

Bellwood, O. (1998). The phylogeny of box crab genera (Crustacea: Brachyura: Calappidae) with notes on their fossil record, biogeography and depth distribution. Journal of the Zoological Society of London, 244, 459-471.

Beurlen, K. (1958). Contribuição à paleontologia do estado do Pará. Boletim do Миseu Paraense Emilio Goeldi, 5, 1-49.

Bourrouilh-Le Jan, F. G., \& Hottinger, L. C. (1988). Occurrence of rhodoliths in the tropical Pacific - a consequence of MidMiocene paleo-oceanographic change. Sedimentary Geology, 60, 355-367.

Brito, I. M. (1971). Contribuição ao conhecimento dos Crustáceos decápodes da Formação Pirabas. I - Brachyura Brachyrhyncha. Anais da Academia Brasileira de Ciências, 43, 489-498.

Brito, I. M. (1972). Contribuição ao conhecimento dos Crustáceos decápodes da Formação Pirabas. II - Brachyura Ocypodidae. Anais da Academia Brasileira de Ciências, 44(1), 95-98.

Brito, I. M. (1977). Novas ocorrências de cirrípedes balanomorfos na Formação Pirabas, Mioceno Inferior do Estado do Pará. Anais da Academia Brasileira de Ciências, 49(3), 465-468.

Brito, I. M. (1993). O aspecto moderno da carcinofauna da Formação Pirabas, Oligoceno-Mioceno do estado do Pará. Anais da Academia Brasileira de Ciências, 65, 57-61.

Bush, M. B., \& Weng, M. B. (2006). Introducing a new (freeware) tool for palynology. Journal of Biogeography, 34, 377-380.

Carter, R. M. (1998). Two models: global sea-level change and sequence stratigraphic architecture. Sedimentary Geology, 122, 23-36.

Colinvaux, P. A., De Oliveira, P. E., \& Patiño, J. E. M. (1999). Amazon Pollen Manual and Atlas - Manual e Atlas Palinológico da Amazônia (p. 332). Amsterdam: Hardwood Academic.

Cookson, I. C., \& Dettmann, M. E. (1958). Some trilete spores from upper Mesozoic deposits in the Eastern Australian region. Proceedings of the Royal Society - Victoria, 70, 95-128.

Cookson, I. C., \& Dettmann, M. E. (1959). On Schizosporis, a new form genus from Australian Cretaceous deposits. Micropalaeontology, 5(2), 213-216.

Costa, J. B. S., Bemerguy, R. L., Hasui, Y., Borges, M. S., Ferreira, J. R. C. R. P., Bezerra, P. E. L., et al. (1996). Neotectônica da Região Amazônica: aspectos tectônicos, geomorfológicos e deposicionais. Geonomos, 4, 23-44.

Dana, J. D. (1852). Macroura. Conspectus Crustaceorum \& Conspectus of the Crustacea of the Exploring Expedition under Capt. C. Wilkes, U.S.N. Proceedings of the Academy of Natural Sciences of Philadelphia, 7, 175-177.

De Grave, S., Pentcheff, N. D., Ahyong, S. T., Chan, T.-Y., Crandall, K. A., Dworschak, P. C., et al. (2009). A classification of living and fossil genera of decapod Crustaceans. Raffles Bulletin of Zoology, 21, 1-109.

Dettmann, M. E. (1963). Upper Mesozoic microforas from southeastern Australia. Proceedings of the Royal Society - Victoria, $77,1-148$.

Dueñas, H. (1980). Palynology of Oligocene-Miocene strata of borehole Q-E-22, Planeta Rica, Northern Colombia. Review of Palaeobotany and Palynology, 10, 318-328.

Fernandes, J. M. G. (1984). Paleoecologia da Formação Pirabas, Estado do Pará. Anais do $33^{\circ}$ Congresso Brasileiro de Geologia, $1,330-340$.
Fernandes, J. M. G., \& Távora, V. A. (1990). Estudo dos Foraminíferos da Formação Pirabas procedentes do furo CB- UFPa-P1 (85), município de Capanema, Estado do Pará. Anais do $36^{\circ}$ Congresso Brasilerio de Geologia, 1, 470-475.

Ferreira, C. S., Macedo, A. C. M. \& Assis, J. F. P. (1978). A Formação Pirabas no Estado do Pará. Novo registro de subsuperfície: Belém (Furo 4be-01-Pa, Cprm). Anais da Academia Brasileira de Ciências, 50(3), 427.

Figueiredo, J., Hoorn, C., van der Ven, P., \& Soares, E. (2009). Late Miocene onset of the Amazon River and the Amazon deep-sea fan: evidence from the Foz do Amazonas Basin. Geology, 37, 619-622.

Frederiksen, N. O., Carr, D. R., \& Lowe, G. D. (1983). Middle Eocene palynomorphs from San Diego, California. Part I. Introduction, spores and gymnosperm pollen. AASP Contribution Series, 12, 1-31.

Germeraad, J. H., Hopping, C. A., \& Muller, J. (1968). Palynology of tertiary sediments from tropical areas. Review of Palaeobotany and Palynology, 6, 189-348.

Góes, A. M., Rossetti, D. F., Nogueira, A. C. R., \& Toledo, P. M. (1990). Modelo deposicional preliminar da Formação Pirabas no nordeste do Estado do Pará. Boletim do Museu Paraense Emílio Goeldi - Ciências da Terra, 2, 3-15.

Gorini, M. A., \& Bryan, G. M. (1976). The tectonic fabric of the equatorial Atlantic and adjoining continental margins: Gulf of Guinea to northeastern Brazil. Anais da Academia Brasileira de Ciências, 48, 101-119.

Grimm, E. (2004). TGView version 2.0.2 (computer software). Illinois State Museum.

Haan, W. de. (1833-1850). Crustacea. In P. F. de Siebold (Ed.), Fauna Japonica sive Descriptio Animalium, quae in Itinere per Japoniam, Jussu et Auspiciis Superiorum, qui Summum in India Batava Imperium Tenent, Suscepto, Annis 1823-1830 Collegit, Notis, Observationibus et Adumbrationibus Illustravit. J. Muller et Co., Lugduni-Batavorum ( $1 / 4$ Leiden).

Halfar, J., \& Mutti, M. (2005). Global dominance of coralline redalgal facies: A response to Miocene oceanographic events. Geology, 33, 481-484.

Haq, B. V, Vali, P. R. \& Hardenhol, J. (1988). Mesozoic and Cenozoic chronostratigraphy and eustatic cycles. In C. K. Wilgus, B. Hastings, C. A. Ross, H. Posamentier, J. Wagoner \& C. G. St. C. Kendall, (Eds.) Sea level changes-an integrated approach (Vol. 42, pp. 71-108). Tulsa: Society of Economic Paleontologists and Mineralogists.

Harris, W. K. (1965). Basal Tertiary microfloras from the Princeton area, Victoria. Palaeontologica, 15, 75-116.

Hesse, M., Halbritter, H., Zetter, R., Weber, M., Buchner, R., FroschRadivo, A., et al. (2008). Pollen terminology: An illustrated handbook (p. 264). New York: Springer.

Hoorn, C. (1993). Marine incursions and the influence of Andean tectonics on the Miocene depositional history of northwestern Amazonia: Results of a palynostratigraphic study. Palaeogeography, Palaeoclimatology, Palaeoecology, 105, 267-309.

Hoorn, C. (1994a). An environmental reconstruction of the palaeoAmazon River system (Middle-Late Miocene, NW Amazonia). Palaeogeography, Palaeoclimatology, Palaeoecology, 112, 187-238.

Hoorn, C. (1994b). Fluvial palaeoenvironments in the intracratonic Amazonas Basin (E arly Miocene-early Middle Miocene, Colombia). Palaeogeography, Palaeoclimatology, Palaeoecology, 109, 1-54.

Hoorn, C., Guerrero, J., Sarmiento, G. A., \& Lorente, M. A. (1995). Andean tectonics as a cause for changing drainage patterns in Miocene northern South America. Geology, 23, 237-240.

Hoorn, C., Wesselingh, F. P., Ter Steege, H., Bermudez, M. A., Mora, A., Sevink, J., et al. (2010). Amazonia through time: Andean 
uplift, climate change, landscape evolution, and biodiversity. Science, 330, 927-931.

Horton, B. K., Parra, M., Saylor, J. E., Nie, J., Mora, A., Torres, V., et al. (2010). Resolving uplift of the northern Andes using detrital zircon age signatures. The Geological Society of America (GSA Today), 20(7), 4-6.

Ibrahim, A. (1933). Sporenformen des Aegirhorizonts des RuhrReviers. Technische Hochschule zu Berlin, Dissertation, pp. 1-46.

Iversen, J., \& Troels-Smith, J. (1950). Pollen Morphologische Funktionen und Typen. Series B - Geological Survey of Denmark, 43(8), 1-54.

Jaramillo, C. A., \& Dilcher, D. L. (2001). Middle Paleogene palynology of Central Colombia, South America: A study of pollen and spores from tropical latitudes. Palaeontographica B, 258, 87-213.

Johns, W. E., Lee, T. N., Beardsley, R. C., Candela, J., Limeburner R. \& Castro B. (1998). Annual cycle and variability of the North Brazil Current. Journal of Physical Oceanography, 28(1), 103-128.

Johns, W. E., Townsend, T. L., Fratantoni, D. M., \& Wilson, W. D. (2002). On the Atlantic inflow to the Caribbean Sea. Deep-Sea Research Part I, 49, 211-243.

Johnson, K. G., Sánchez-Villagra, M. R., \& Aguilera, O. (2009). The Oligocene-Miocene transition on coral reefs in the Falcón Basin (NW Venezuela). Palaios, 24, 59-69.

Karasawa, H., Schweitzer, C. E., \& Feldmann, R. M. (2008). Revision of Portunoidea Rafinesque, 1815 (Decapoda: Brachyura) with emphasis on the fossil genera and families. Journal of Crustacean Biology, 28(1), 82-127.

Kominz, M. A., Browning, J. V., Miller, K. G., Sugarman, P. J., Mizintseva, S., \& Scotese, C. R. (2008). Late Cretaceous to Miocene sea-level estimates from the New Jersey and Delaware coastal plain coreholes: An error analysis. Basin Research, 20, 211-226.

Krutzsch, W. (1959). Einige neue Formgattugen und-arten von Sporen und Pollen aus der mitteleuropaischen Oberkreide und dem Tertiar. Palaeontographica, 105, 125-157.

Lalor, A. M., \& Távora, V. (2006). Novos elementos da coralinofauna da formação Pirabas (Mioceno inferior), estado do Pará. Geociências, 25(2), 187-195.

Latreille, P. A. (1802-1803). Histoire naturelle, générale et particuliere, des crustacés et des insects (Vol. 3). Paris: F. DuFart.

Legoux, O. (1978). Quelques espèces e pollen caractéristiques du Neogene du Nigeria. Bulletin Centre Recherche. ExplorationProduction Elf-Aquitaine, 2(2), 265-317.

Leite, F. P. R., Oliveira, M. E. B., Arai, M., \& Truckenbrodt, W. (1997a). Palinoestratigrafia da Formação Pirabas e Grupo Barreiras, Mioceno do nordeste do estado do Pará, Brasil. Revista da Universidade de Guarulhos, 2, 141-147.

Leite, F. P. R., Oliveira, M. E. B., Oliveira, P. E., Silvestre-Capelato, M. S., Arai, M., \& Truckenbrodt, W. (1997b). Palinofloras miocenas da Formação Pirabas e Grupo Barreiras, na Região Bragantina, Estado do Pará, Brasil. Revista da Universidade de Guarulhos, 2, 128-140.

Lorente, M.A. (1986). Palynology and palynofacies of the upper Tertiary in Venezuela. (Dissertationes Botanicae, Vol. 99). Berlin-Stuttgart: Lubrecht \& Cramer Ltd.

Manning, R. B., \& Felder, D. L. (1991). Revision of the American Callianassidae (Crustacea: Decapoda: Thalassinidea). Proceedings of the Biological Society of Washington, 104(4), 764-792.

Martins-Neto, R. G. (2001). Review of some Crustacea (Isopoda and Decapoda) from Brazilian deposits (Paleozoic, Mesozoic and Cenozoic) with descriptions of new taxa. Acta Geologica Leopoldensia, 24(52/53), 237-254.

Martins-Neto, R. G. (2005). Estágio atual da paleoartropodologia brasileira: hexápodes, miriápodes, crustáceos (isopoda, decapoda,
Eucrustacea e Copepoda) e Quelicerados. Arquivos do Museu Nacional, 63(3), 471-494.

Martins-Neto, R. G., \& Dias-Júnior, S. C. (2007). The Brazilian paleodecapod fauna: state of knowledge. Memorie della Società Italiana di Scienze Naturali e del Museo Civico di Storia Naturale di Milano, 35(2), 76-78.

Maury, C. J. (1925). Fósseis terciários do Brasil com descrição de novas formas Cretáceas. Serviço Geológica e Mineralógico do Brasil - Monografia, 4, 1-665.

Miall, A. D. (1978). Facies types and vertical profile models in braided river deposits: a summary. In A. D. Miall (Ed.), Fluvial sedimentology (pp. 597-604). Calgary: Canadian Society of Petroleum Geologists.

Milne Edwards, A. (1873). Pages 8-9, pi. 4. In M. Le Comte R. de Bouille, Paleontologie de Biarritz et de quelques autres localities des Basses-Pyrenees. C. R. Trav. Congr. Sci. Fr., sess. 39e, Pau, t. 4 , f. 3, pt. 1.

Milne Edwards, A. (1881). Note sur quelques Crustacés fossiles des environs de Biarritz. Annales des Sciences Geologiques - Paris, 11(1-8), 21-22.

Miner, E. L. (1935). Paleobotanical examinations of Cretaceous and Tertiary coals. American Midland Naturalist, 16, 585-625.

Muller, J., Giacomo, E., \& Van Erve, A. W. (1987). A palynological zonation for the Cretaceous, Tertiary and Quaternary of northern South America. American Association of Stratigraphic Palynologists, contribution series, 19, 7-76.

Nakoman, E. (1965). Description d'un noveau genre de for, Corsinipollenites. Annales de la Societe Geologique du Nord, $85,155-158$.

Nakoman, E. (1966). Contribution à l' étude palynologique des formations tertiaires du Bassin de Thrace. Annales de la Societé Géologique du Nord, 46, 65-107.

Nyborg, T. G., Berglund, R. E., \& Goedert, J. L. (2003). A new crab from the late Eocene Hoko River Formation, Olympic Peninsula, Washington: the earliest record of Euphylax (Decapoda: Portunidae). Journal of Paleontology, 77, 323-330.

Paula-Couto, C. (1967). Contribuição à paleontologia do estado de Pará. Um sirênio na formação Pirabas. Atas do lo Simpósio sobre a Biota Amazônica, 1, 345-357.

Pérez-Ruzafa, A., Alvarado, J. J., Solís-Marín, F. A., Hernández, J. C., Morata, A., Marcos, C., et al. (2012). Latin America echinoderm biodiversity and biogeography: Patterns and affinities. In J. J. Alvarado \& F. A. Solís-Marín (Eds.), Echinoderm research and diversity in Latin America (pp. 511-541). New York: Springer.

Petri, S. (1954). Foraminíferos fósseis da Bacia de Marajó. Boletim da Faculdade de Filosofia, Ciências e Letras da Universidade de São Paulo (Geologia), 134(11), 1-70.

Petri, S. (1957). Foraminíferos Miocênicos da Formação Pirabas. Boletim da Faculdade de Filosofia. Ciências e Letras da Universidade de São Paulo (Geologia), 216, 1-172.

Playford, G. (1982). Neogene palynomorphs from the Huon Peninsula, Papua New Guinea. Palynology, 6, 29-54.

Potonié, R. (1951). Revision stratigraphisch wichtiger Sporomorphen des mitteleuropaischen Tertiars. Palaeontographica B, 9, 131-151.

Potonié, R. (1956). Synopsis der Gattungen der sporae dispersae. I Teil: Sporites. Beihefte zum Geologischen Jahrbuch, 23, 1-103.

Potonié, R., \& Gelletich, J. (1933). Ueber Pteridophyten-Sporen einer eozänen Braunkohle aus Dorog in Ungarn: Gesellschaft Naturforschender Freunde zu Berlin. Sitzungsberichte, 33, 517-528.

Potonié, R., \& Kremp, G. O. W. (1954). Die Gattungen der palãozoischen Sporae dispersae und ihre Stratigraphie. Geologisches Jahrbuch, 69, 111-194.

Punt, W., Hoen, P. P., Blackmore, S., Nilsson, S., \& Le Thomas, A. (2007). Glossary of pollen and spore terminology. Review of Palaeobotany and Palynology, 143, 1-81. 
Rafinesque, C. S. (1815). Analyse de la nature, ou tableau de l'univers et des corps organisés. Palermo: L'Imprimerie de Jean Barravecchia, p. 224.

Rathbun M. J. (1924). Fossil crabs from the Republic of Haiti. Proceedings of the Biological Society of Washington, 63, 1-6, pls. 1-2.

Regali, M. S. P., Uesugui, N., \& Santos, A. S. (1974). Palinologia dos sedimentos meso-cenozóicos do Brasil (I). Boletim Técnico da Petrobrás, 13(3), 177-191.

Rich, F. J., Kuehn, D., \& Davies, T. D. (1982). The paleoecological significance of Ovoidites. Palynology, 6, 19-28.

Rossetti, D. F. (2001). Late Cenozoic sedimentary in northeastern Pará, Brazil, within the context of sea-level changes. Journal of South America Earth Science, 14, 77-89.

Rossetti, D. F. (2006). Evolução sedimentar Miocênica nos Estados do Pará e Maranhão. Revista do Instituto de Geociências, 6, 7-18.

Rossetti, D. F., \& Góes, A. M. (2004). Geologia. In D. F. Rossetti \& A. M. Góes (Eds.), O Néogeno da Amazônia Oriental (pp. 13-52). Belém: Editora MPEG.

Rossetti, D. F., \& Santos, A. E. A., Jr. (2004). Facies architecture in a tectonically-influenced estuarine incised valley fill of Miocene age, Northern Brazil. Journal of South America Earth Sciences, 17, 267-284.

Roubik, D. W., \& Moreno, J. E. (1991). Pollen and spores of Barro Colorado Island (p. 268). St. Louis: Missouri Botanical Garden.

Sah, S. C. D. (1967). Palynology of an upper Neogene profile from Rusizi Valley (Burundi). Musée Royal de l'Afrique Centrale, Tervuren, Belgique, Annales, Series, 8(57), 1-173.

Sakai, K. (1988). A new genus and five new species of Callianassidae (Crustacea: Decapoda: Thalassinidea) from northern Australia. The Beagle, Records of the Northern Territory Museum of Arts and Sciences, 5, 51-69.

Salard-Cheboldaeff, M. (1990). Intertropical African palynostratigraphy from Cretaceous to late quaternary times. Journal of African Earth Sciences, 11, 1-24.

Santos, M. E. C. M. (1958). Equinóides miocênicos da Formação Pirabas. Boletim da Divisão de Geologia e Mineralogia, 179, $1-24$.

Santos, M. E. C. M. (1967). Equinóides miocênicos da Formação Pirabas. Atas do 10 Simpósio sobre a Biota Amazônica, 1, 407-410.

Santos, A. S., Carvalho, M. A., Santos, T. M., \& Freitas, A. G. (2005). Caracterização da flora de montanha Quaternária com base em estudos palinológicos da Bacia da foz do Amazonas, Brasil. Arquivos do Museu Nacional, 63, 425-436.

Santos, D. B., Garcia, M. J., Saad, A. R., \& Bistrichi, C. A. (2010). Palinoestratigrafia da Formação Itaquaquecetuba, Bacia de São Paulo, Brasil. Revista Brasileira de Paleontologia, 13(3), 205-220.

Santos, R. S., \& Salgado, M. S. (1971). Contribuição à paleontologia do Estado do Pará. Novos restos de peixes da Formação Pirabas. Boletim do Museu Paraense Emílio Goeldi, 16, 1-13.

Santos, R. S., \& Travassos, S. (1960). Contribuição à paleontologia do Estado do Pará. Peixes fósseis da Formação Pirabas. Divisão de Geologia e Mineralogia, Departamento Nacional da Produção Mineral, 16, 1-35.

Schweitzer, C. E., Feldmann, R. M., Garassino, A., Karasawa, H., \& Schweigert, G. (2009). Systematic list of fossil decapod crustacean species. Brill, Leiden, Crustaceana Monographs, 10, $1-221$.

Schweitzer, C. E., Iturralde-Vinent, M., Hetler, J. L., \& Velez-Juarbe, J. (2006). Oligocene and Miocene decapods (Thalassinidea and Brachyura) from the Caribbean. Annals of Carnegie Museum, 75(2), 111-136.
Shephard, G. E., Müller, R. D., Liu, L., \& Gurnis, M. (2010). Miocene drainage reversal of the Amazon River driven by plate-mantle interaction. Nature Geoscience, 3, 870-875.

Silva-Caminha, S. A. F., Jaramillo, C. A. \& Absy, M. L. (2010). Neogene palynology of the Solimões Basin, Brazilian Amazonia. Palaeontographica Abteilung B: Palaeobotany - Palaeophytology, 283, 1-67.

Singh, C. (1964). Microflora of the Lower Cretaceous Mannville Group, east-central Alberta. Research Council of Alberta Bulletin, 15, 1-239.

Stimpson, W. (1862-1860). Notes on North American Crustacea, in the Museum of the Smithsonian Institution. No. II. Annals of the Lyceum of Natural History, 7, 176-246.

Szatmari, P., Françolin, J. B. L., Zanotto, O., \& Wolff, S. (1987). Evolução tectônica da margem equatorial brasileira. Revista Brasileira de Geociências, 17, 180-188.

Távora, V., \& Fernandes, J. M. (1999). Estúdio de los foraminíferos de la Formación Pirabas (Mioceno Inferior), Estado de Pará, Brasil, y su correlación con faunas del Caribe. Revista Geológica de America Central, 22, 63-74.

Távora, V. A., Silveira, E. S. F., \& Milhomem Neto, J. M. (2007). Mina B-17, Capanema, PA - Expressivo Registro de uma Paleolaguna do Cenozóico Brasileiro. In: M. Winge, C. Schobbenhaus, M. Berbert-Born, E. T. Queiroz, D. A. Campos, C. R. G. Souza, \& A. C. S. Fernandes, (Eds.), Sítios Geológicos e Paleontológicos do Brasil (pp. 18). Brasília: CPRM. http://www. unb.br/ig/sigep/sitio121/sitio121.pdf.

Távora, V., Santos, A.A., \& Araujo, R.N. (2010). Localidades fossilíferas da Formação Pirabas (Mioceno Inferior). Boletim do Museu Paraense Emilio Goeldi - Ciências Naturais, 5(2), 207-224.

Thiegart, F. (1940). Die mikropaläontologie als pollenanalyse im dienst der Braunkohlenforschung. Brennstoff-Geologie Sehr, 13, 50.

Toledo, P. M. (1989). Sobre novos achados de sirênios (Sirenotherium Pirabense, Paula Couto, 1967) na Formação Pirabas (Pará, Brasil). Boletim do Museu Paraense Emílio Goeldi, 1, 5-10.

Toledo, P. M., \& Domning, D. P. (1989). Fossil Sirenia (Mammalia: Dugongidae) from the Pirabas Formation (Early Miocene), northern Brazil. Boletim do Museu Paraense Emílio Goeldi, série Ciências da Terra, 1(2), 119-146.

Traverse, A. (2008). Paleopalynology. Dordrecht: Springer.

Uesugui, N. (1979). Palinologia: técnicas de tratamento de amostras. Boletim Técnico Petrobrás, 22, 229-240.

Van der Hammen, T. (1956a). Description of some genera and species of fossil pollen and spores. Boletín Geológico, 4, 103-109.

Van der Hammen, T. (1956b). A palynological systematic nomenclature. Boletín Geológico, 4, 63-101.

Van der Hammen, T., \& Garcia, C. (1966). The Paleocene pollen flora of Colombia. Leidse Geologische Mededelingen, 35, 105-114.

Van der Hammen, T., \& Wymstra, T. A. (1964). A palynological study on the Tertiary and Upper Cretaceous of British Guiana. Leidse Geologische Mededelingen, 30, 183-241.

Van Geel, B., \& Van der Hammen, T. (1978). Zygnemataceae in Quaternary, Colombian sediments. Review of Palaeobotany and Palynology, 25, 377-392.

Van Hoeken-Klinkenberg, P. M. J. (1964). A palynological investigation of some Upper Cretaceous sediments in Nigeria. Pollen et Spores, 6, 209-231.

Van Hoeken-Klinkenberg, P. M. J. (1966). Maastrichtian, Paleocene and Eocene pollen and spores from Nigeria. Leidse Geologische Mededelingen, 38, 37-48.

Vega, F. J., Nyborg, T., Coutiño, M. A., Solé, J., \& HernándezMonzón, O. (2009). Neogene Crustacea from southeastern Mexico. Bulletin of the Mizunami Fossil Museum, 35, 51-69. 
Walker, R. G. (1992). Facies, facies models and modern stratigraphic concepts. In R. G. Walker \& N. P. James (Eds.), Facies modelsresponse to sea level change (pp. 1-14). Ontario: Geological Association of Canada.

Weber, F. (1795). Nomenclator entomologicus secundum Entomologiam Systematicum ill. Fabricii, adjectis speciebus recens detectis et varietatibus. In C. E. Bohn, Chilonii \& Hamburgi, viii p $171 \mathrm{pp}$
White, C. A. (1887). Contribuições à Paleontologia do Brasil. Archivos do Museu Nacional, 7, 1-273.

Wilson, L. R., \& Webster, R. M. (1946). Plant microfossils from a Fort Union coal of Montana. American Journal of Botany, 33, 271-278.

Zachos, J., Pagani, M., Sloan, L., Thomas, E., \& Billups, K. (2001). Trends, rhythms, and aberrations in global climate $65 \mathrm{Ma}$ to present. Science, 292, 686-693. 